\title{
Three-dimensional Floquet stability analysis of the wake of a circular cylinder
}

\author{
By DWIGHT BARKLEY AND RONALD D. HENDERSON ${ }^{2}$ \\ ${ }^{1}$ Nonlinear Systems Laboratory, Mathematics Institute, \\ University of Warwick, Coventry, CV4 7AL, UK \\ ${ }^{2}$ Aeronautics and Applied Mathematics, \\ California Institute of Technology, Pasadena, CA 91125, USA
}

(Received 20 March 1995 and in revised form 16 April 1996)

Results are reported from a highly accurate, global numerical stability analysis of the periodic wake of a circular cylinder for Reynolds numbers between 140 and 300 . The analysis shows that the two-dimensional wake becomes (absolutely) linearly unstable to three-dimensional perturbations at a critical Reynolds number of $188.5 \pm 1.0$. The critical spanwise wavelength is $3.96 \pm 0.02$ diameters and the critical Floquet mode corresponds to a 'Mode A' instability. At Reynolds number 259 the two-dimensional wake becomes linearly unstable to a second branch of modes with wavelength 0.822 diameters at onset. Stability spectra and corresponding neutral stability curves are presented for Reynolds numbers up to 300 .

\section{Introduction}

The aim of this paper is to quantify an important step in the sequence of instabilities leading to turbulence in the wake of a circular cylinder. Specifically, we present results from a highly accurate, global stability analysis of the two-dimensional von Kármán vortex street and identify precisely the secondary linear instability leading to threedimensionality in this flow.

The system considered is an infinitely long circular cylinder placed perpendicular to an otherwise uniform open flow. The sole parameter for this system is then the Reynolds number: $\operatorname{Re} \equiv U_{\infty} d / v$, where $U_{\infty}$ is the free-stream velocity, $d$ is the cylinder diameter, and $v$ is the kinematic viscosity. Changes in the wake dynamics as a function of $R e$ are typically quantified using either force or shedding frequency measurements. In non-dimensional form, the vortex shedding frequency $f$ is known as the Strouhal number: $S t \equiv f d / U_{\infty}$. Figure 1 shows a plot of Strouhal number over the range of Reynolds number that includes the first few important wake instabilities.

For low values of the Reynolds number, the flow is steady and two-dimensional. At $R e_{1} \approx 46$ the flow undergoes a supercritical Hopf bifurcation (the primary instability) that leads to a two-dimensional oscillatory flow: the well-known von Kármán vortex street. Both numerical stability calculations by Jackson (1987) and experiments by Mathis, Provansal \& Boyer (1987) confirm the critical values for this instability. For a range of $R e$ above this bifurcation, the wake is laminar and perfectly time-periodic with a unique relation between Reynolds number and vortex shedding frequency. What is not well understood is the subsequent sequence of bifurcations leading to turbulence in this system. It is known that there are no further two-dimensional 


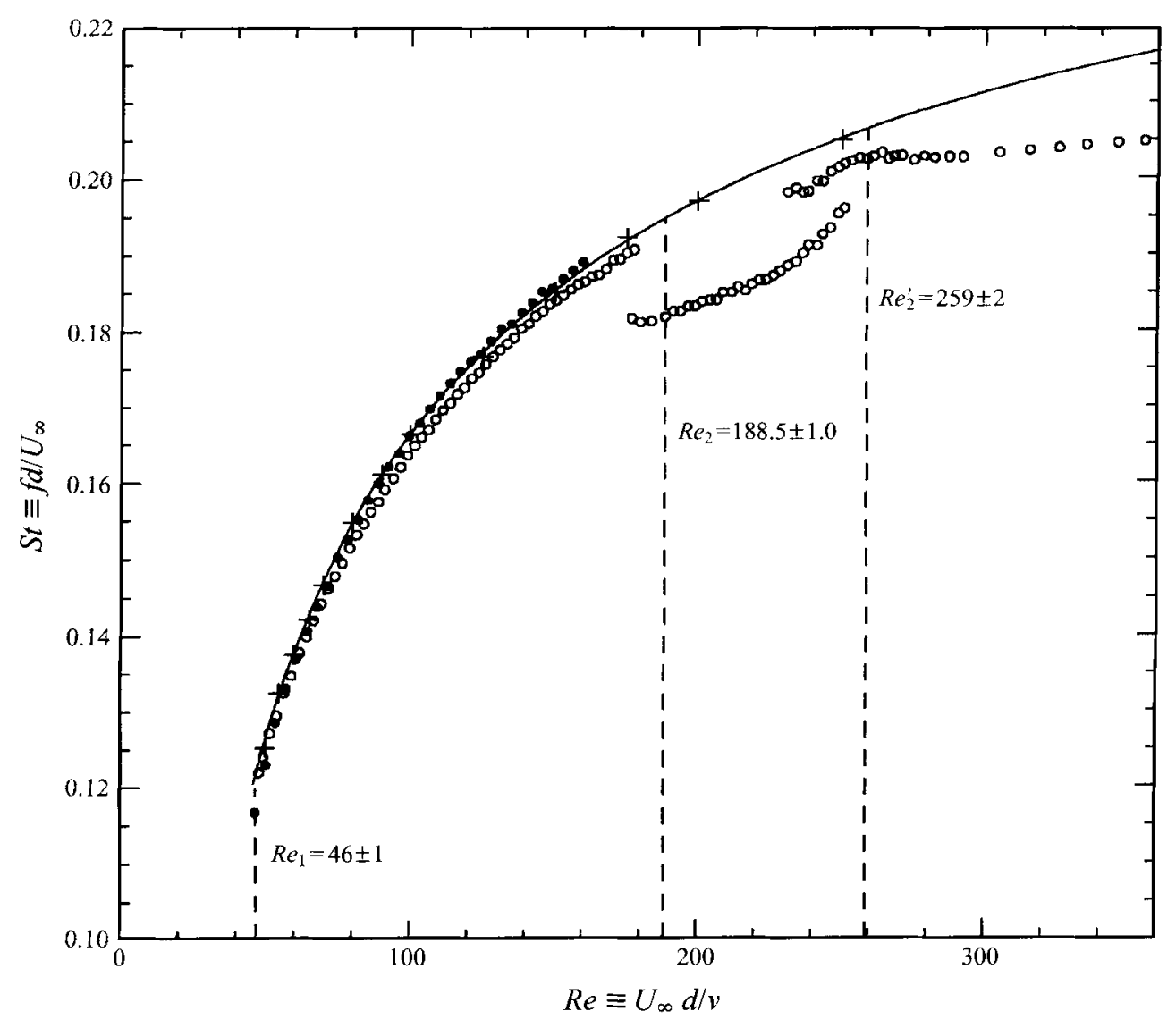

Figure 1. Variation of Strouhal number with Reynolds number for the cylinder wake, from experimental measurements and computer simulations of two-dimensional flow: o, Williamson (1989); •, Hammache \& Gharib (1991); +, flow calculations from the current study (the solid line is a curve fit to the simulation data for $R e$ up to 1000). The first few important wake instabilities are labelled: $R e_{1}$ (the primary instability) is a Hopf bifurcation to a two-dimensional time-periodic wake; $R e_{2}$ (the secondary instability) is the point at which the two-dimensional wake first becomes linearly unstable to three-dimensional disturbances; $R e_{2}^{\prime}$ is a point of further three-dimensional instability described in $\S 3 . R e_{2}$ and $R e_{2}^{\prime}$ were determined as part of the current study.

bifurcations in the flow dynamics prior to the secondary instability at $\operatorname{Re}_{2}$, the critical point for the bifurcation giving rise to three-dimensionality. However, exact details of the secondary instability and subsequent transitions leading to turbulence are not clear owing to several conspiring factors.

On the experimental side, there is considerable scatter in the observed values of Reynolds number for the transition to three-dimensionality, with values of $R e_{2}$ ranging from about 140 to 190 . Roshko (1993) and Williamson (1996b) review many of the recent experimental findings. Factors such as cylinder roughness, free-stream turbulence amplitude, blockage, and end effects all tend to shift transition of the laminar wake to lower values of $R e$. Even in facilities with seemingly large cylinder aspect ratios, end conditions have a dramatic effect across the entire span. This has made it difficult to separate the effects of extrinsic three-dimensionality present in experiments from intrinsic three-dimensionality that arises from the stability properties of the natural wake. By controlling end ef- 
fects, it is possible to produce a relatively good approximation to flow past an infinitely long cylinder in the laboratory as demonstrated with various techniques by Williamson (1989) and Hammache \& Gharib (1991). The data shown in figure 1 are for such conditions. Three-dimensionality first appears in these measurements at $R e \approx 180$, but precise details of the transition cannot be determined from the experiments.

On the computational side, direct numerical simulation of transition in the wake is a demanding calculation that has only become practical with recent advances in algorithms and computer performance. Karniadakis \& Triantafyllou (1992) and Tomboulides, Triantafyllou \& Karniadakis (1992) conducted the earliest computational studies of the three-dimensional cylinder wake, finding that the two-dimensional wake is unstable to three-dimensional disturbances at $R e \approx 200$. In these calculations they observed a period-doubling bifurcation at $R e \approx 300$ and proposed that the wake might follow a period-doubling cascade leading to turbulence. Recently, a number of additional computational studies have appeared which examine the threedimensional flow that develops over this same range of Reynolds number (Zhang et al. 1995; Mittal \& Balachandar 1995; Thompson, Hourigan \& Sheridan 1996). However, in none of these numerical studies was an attempt made to determine the onset of the secondary instability precisely because of the computational expense of such calculations using direct simulations. Noack, König \& Eckelmann (1993) and Noack \& Eckelmann $(1994 a, b)$ have performed a Floquet stability analysis, similar in spirit to the one reported here, using a Galerkin projection onto a moderate number of analytically determined modes. They find a bifurcation leading to threedimensionality that is qualitatively, though not quantitatively, in agreement with our computations.

We report here fully resolved Floquet stability calculations of the time-periodic wake of the circular cylinder throughout the transition regime $140 \leqslant R e \leqslant 300$. Because our goal is an accurate stability calculation for an infinitely long cylinder in an open flow, a significant portion of the paper is devoted to numerical convergence studies. The stability computations reported provide the first direct and highly accurate determination of the critical Reynolds number and the critical spanwise wavenumber for the secondary instability of the von Kármán wake, and the computations allow a direct visualization of the structure of the critical eigenmode (Floquet mode) that drives this instability. In addition, our calculations provide stability spectra throughout the transition regime and thus allow an accurate determination of the bands of unstable spanwise wavenumbers above the onset of three-dimensionality. We show that our results are supported by experimental flow visualization and numerous measurements at Reynolds numbers slightly beyond transition. However, because the onset of three-dimensionality in experiments is at present somewhat unclear, our primary concern is to present accurate computations directly from the Navier-Stokes equations for which we can provide definitive answers.

\section{Computational methods}

Our numerical computations consist of two parts. First we obtain solutions for flow past a circular cylinder from time-dependent simulations of the Navier-Stokes equations on two-dimensional domains. Next we determine the stability of these solutions via Floquet stability analysis. 


\subsection{Time-dependent simulations}

The fluid flow is governed by the incompressible Navier-Stokes equations:

$$
\begin{gathered}
\frac{\partial \boldsymbol{u}}{\partial t}=-\mathbf{N}(\boldsymbol{u})-\frac{1}{\rho} \nabla p+\frac{1}{R e} \nabla^{2} \boldsymbol{u} \text { in } \Omega, \\
\nabla \cdot \boldsymbol{u}=0 \quad \text { in } \Omega,
\end{gathered}
$$

where $u \equiv(u, v, w)$ is the velocity field, $p$ is the static pressure, $\rho$ is the fluid density, $R e$ is the Reynolds number, and $\Omega$ is the computational domain. Without loss of generality we take the numerical value of $\rho=1$ since this simply sets the scale for $p$. $\mathbf{N}(\boldsymbol{u})$ represents the nonlinear advection term:

$$
\mathbf{N}(\boldsymbol{u}) \equiv(\boldsymbol{u} \cdot \nabla) \boldsymbol{u}
$$

Equations (2.1) are written in non-dimensional form: lengths are scaled by the cylinder diameter, $d$, and velocities are scaled by $U_{\infty}$, the magnitude of the free-stream velocity, i.e. $\boldsymbol{u}$ at infinity.

Time-dependent simulations based on these equations in two dimensions $(w \equiv 0$, $\partial / \partial z \equiv 0$ ) are carried out using a spectral element program (Prism, Henderson 1994). In the spectral element method, the domain $\Omega$ is represented by a mesh of $K$ elements and within each element both the geometry and the solution variables, in this case velocity and pressure, are represented using $N$ th-order tensor-product polynomial expansions. We use the Gauss-Lobatto Legendre polynomials as a basis for the numerical solution. The meshes used in our study are shown in figure 2 and are discussed in detail in $\S 2.3$. A high-order time-splitting scheme was used to integrate (2.1). The splitting scheme replaces this system with an advection equation and a sequence of elliptic Helmholtz-type equations, each of which can be discretized using a weak Galerkin formulation. We solved the resulting linear systems directly using a static condensation algorithm tailored to the unique structure of spectral element matrices; all arithmetic was carried out in 64-bit precision. Henderson \& Karniadakis (1995) discuss details of the method and solution techniques, along with various validation studies for the particular code employed here.

Boundary conditions for the velocity and pressure fields are required along the exterior of $\Omega$, which can be divided into three sections: $\Gamma_{\infty}$ is composed of the left, upper, and lower boundaries; $\Gamma_{o}$ is composed of the right (outflow) boundary; and $\Gamma_{b}$ is the surface of the cylinder. Boundary conditions at $\Gamma_{\infty}$ and $\Gamma_{o}$ define the flow far from the cylinder. Along $\Gamma_{\infty}$ we enforce uniform flow $(u=1, v=0)$, while along $\Gamma_{o}$ we use a standard outflow boundary condition for the pressure and velocity:

$$
p=0, \quad \partial u / \partial x=0, \quad \partial v / \partial x=0 .
$$

At the surface of the cylinder, $\Gamma_{b}$, the flow satisfies no-slip boundary conditions. Boundary conditions for the pressure, other than along $\Gamma_{o}$, can be generated by requiring the computed flow to satisfy the normal component of the momentum equation along the boundaries. This is the high-order Neumann boundary condition suggested by Karniadakis, Israeli \& Orszag (1991) and shown to control divergence errors normally associated with splitting methods for incompressible flows.

Wake flows for our stability calculations were obtained from simulations with Reynolds numbers in the range $140 \leqslant R e \leqslant 300$. In all cases, the simulations were run sufficiently long to obtain asymptotic, time-periodic velocity fields. These periodic solutions were then stored for use in our stability calculations by saving 32 velocity fields (snapshots) equally spaced over one period. The periodic flow can be obtained 
at any time from the 32 velocity fields, via Fourier interpolation in time, to within the overall accuracy of the simulations.

\subsection{Floquet stability analysis}

Let $\boldsymbol{U}(x, y, t)$ be the two-dimensional wake (base flow) of period $T$ whose stability is sought. An infinitesimal three-dimensional perturbation $\boldsymbol{u}^{\prime}(x, y, z, t)$ to this base flow evolves according to the linearized Navier-Stokes equations:

$$
\begin{gathered}
\frac{\partial u^{\prime}}{\partial t}=-\mathbf{D N}\left(u^{\prime}\right)-\frac{1}{\rho} \nabla p^{\prime}+\frac{1}{R e} \nabla^{2} u^{\prime} \text { in } \Omega, \\
\nabla \cdot u^{\prime}=0 \text { in } \Omega,
\end{gathered}
$$

where $p^{\prime}$ is the perturbation to the pressure and $\mathbf{D N}\left(\boldsymbol{u}^{\prime}\right)$ is the linearized advection term:

$$
\mathrm{DN}\left(\boldsymbol{u}^{\prime}\right) \equiv\left(\boldsymbol{u}^{\prime} \cdot \nabla\right) \boldsymbol{U}+(\boldsymbol{U} \cdot \nabla) \boldsymbol{u}^{\prime}
$$

The boundary conditions on the perturbed velocity are: $\boldsymbol{u}^{\prime}=0$ along $\Gamma_{\infty}$ and $\Gamma_{b}$; along $\Gamma_{o}$ we again use an outflow boundary condition as in (2.2). Thus, the perturbed flow $U+u^{\prime}$ satisfies the same boundary conditions as the base flow.

By defining the operator $\mathbf{L}$ so that $\mathbf{L}\left(\boldsymbol{u}^{\prime}\right)$ is the right-hand-side of the linearized equation $(2.3 a)$ subject to the incompressibility constraint $(2.3 b)$, we can write the evolution equation for infinitesimal perturbations compactly as

$$
\frac{\partial \boldsymbol{u}^{\prime}}{\partial t}=\mathbf{L}\left(\boldsymbol{u}^{\prime}\right)
$$

The operator $\mathbf{L}\left(\boldsymbol{u}^{\prime}\right)$ is $T$-periodic because $\mathbf{D N}\left(\boldsymbol{u}^{\prime}\right)$ is, through the base flow $U$, and therefore (2.4) is of Floquet type. Solutions of this equation can be decomposed into a sum of solutions of the form: $\tilde{u}(x, y, z, t) \exp (\sigma t)$, where the $\tilde{u}(x, y, z, t)$ are also $T$-periodic functions. These functions are the Floquet modes of the operator $\mathbf{L}$. The complex numbers $\sigma$ are the Floquet exponents, although for Floquet problems one typically considers instead the Floquet multipliers $\mu \equiv \exp (\sigma T)$.

Stability of the base flow $U$ is determined by the spectrum of multipliers or eigenvalues of the operator $\mathbf{L}$. Floquet multipliers inside the unit circle in the complex plane $(|\mu|<1)$ correspond to exponentially decaying solutions ( $\operatorname{Re} \sigma<0$ ), while multipliers outside the unit circle $(|\mu|>1)$ correspond to exponentially growing solutions $(\operatorname{Re} \sigma>0)$. An instability is signalled by a multiplier crossing the unit circle, and because we consider the full flow field subject to inflow-outflow boundary conditions, these instabilities are global and absolute (Huerre \& Monkewitz 1990).

A further simplification is possible because the system is homogeneous in the spanwise direction $z$. General perturbations to the velocity field can be expressed as the Fourier integral

$$
\boldsymbol{u}^{\prime}(x, y, z, t)=\int_{-\infty}^{\infty} \hat{\boldsymbol{u}}(x, y, \beta, t) \mathrm{e}^{\mathrm{i} \beta z} \mathrm{~d} \beta,
$$

and similarly for $p^{\prime}$. Because equations (2.3) are linear, modes with different $|\beta|$ do not couple. In fact, perturbations of the form:

$$
\left.\begin{array}{l}
\boldsymbol{u}^{\prime}(x, y, z, t)=(\hat{u} \cos \beta z, \hat{v} \cos \beta z, \hat{w} \sin \beta z) \\
p^{\prime}(x, y, z, t)=\hat{p} \cos \beta z
\end{array}\right\},
$$

remain of this form under equation (2.3). Floquet modes $\tilde{u}(x, y, z, t)$ will then necessarily be of this same form. Because the three velocity components $(\hat{u}, \hat{v}, \hat{w})$ and pressure 
$\hat{p}$ depend only on $x, y$, and $t$, the full three-dimensional stability problem at any fixed Reynolds number can be reduced to a one-parameter family of two-dimensional stability problems. To analyse the stability of the von Kármán vortex street we solve the reduced problem by computing the Floquet multipliers, $\mu$, and corresponding modes, $\tilde{\boldsymbol{u}}$, as a function of the two parameters $R e$ and $\beta$.

To find the Floquet modes of the system (2.4) corresponding to multipliers near the unit circle, we use a method similar to that used by Goldhirsch, Orszag \& Maulik (1987), Amon \& Patera (1989), and Mamun \& Tuckerman (1995). This technique is also described by Schatz, Barkley \& Swinney (1995). We first construct an operator representing the evolution of the linear system (2.3), or equivalently (2.4), over one period $T$ :

$$
\boldsymbol{u}_{n+1}^{\prime}=\mathbf{A}\left(\boldsymbol{u}_{n}^{\prime}\right)
$$

where $\boldsymbol{u}_{n}^{\prime}=\boldsymbol{u}^{\prime}\left(x, y, z, t_{0}+n T\right)$ is the perturbation after $n$ periods. The operator $\mathbf{A}$ is then equivalent to the linearized Poincare map associated with the periodic base flow. The eigenvalues $\mu$ of $\mathbf{A}$ are precisely the Floquet multipliers of $\mathbf{L}$ and the eigenfunctions $\tilde{\boldsymbol{u}}\left(x, y, z, t_{0}\right)$ of $\mathbf{A}$ are the Floquet modes at some instant in time $t_{0}$, where $t_{0}$ depends on the phase of the base flow $\boldsymbol{U}$ implicit in the definition of $\mathbf{A}$. Floquet modes $\tilde{\boldsymbol{u}}$ at other times can be found by a further integration of (2.4).

The action of $\mathbf{A}$ on a perturbation is obtained by integrating the linearized equations (2.3) using essentially the same methods as for the full Navier-Stokes equations (2.1). Two principal changes are required. First, the nonlinear operator $N(\boldsymbol{u})$ is replaced by the linear operator $\mathbf{D N}\left(\boldsymbol{u}^{\prime}\right)$. The base flow $\boldsymbol{U}$ appearing in this operator is obtained at each time step by Fourier interpolation in time from the 32 stored velocity fields obtained as described in the previous section. Second, we replace the operator $\nabla$ everywhere in $(2.3)$ by $(\partial / \partial x, \partial / \partial y,-i \beta)$, and compute the three velocity components $(\hat{u}, \hat{v}, \hat{w})$ and pressure $\hat{p}$ on two-dimensional domains.

We use subspace iteration to obtain the eigenvalues of $\mathbf{A}$ with largest magnitude. Watkins (1993) gives a thorough review of similar methods for large eigenvalue problems. The iteration is performed on a Krylov subspace of dimension between 8 and 20, initialized either from a random starting vector or an eigenmode computed at nearby parameter values. The iteration yields converged eigenvalues after 6 to 25 iterations of the operator A. Because the leading eigenvalues are well-separated over the range of $R e$ considered, no preconditioning was necessary to achieve this rate of convergence. The accuracy of the eigenvalues is addressed in the following section.

\subsection{Convergence tests}

Approximation errors in the base flow and stability calculations can be considered separately. For the base flow computations we verified that our results were meshindependent to a high degree of precision and that our two-dimensional wake results reproduced the best experimental data available. For the stability calculations we again checked for mesh independence and in addition estimated the precision of eigenvalues in terms of a calculable residual.

We tested the base flow and Floquet multipliers for sensitivity to domain size and resolution using the family of meshes shown in figure 2 . In the spectral element method one generally follows a p-type finite element approach, first constructing a mesh of elements that gives a good approximation to the flow and then increasing the polynomial order of the local basis functions within each element to produce a converged solution. With the exception of $M_{3}$, each mesh is an exact subset of the largest mesh $M_{6}$, differing only in the three domain size parameters: $l_{i}$ (inflow), $l_{o}$ 

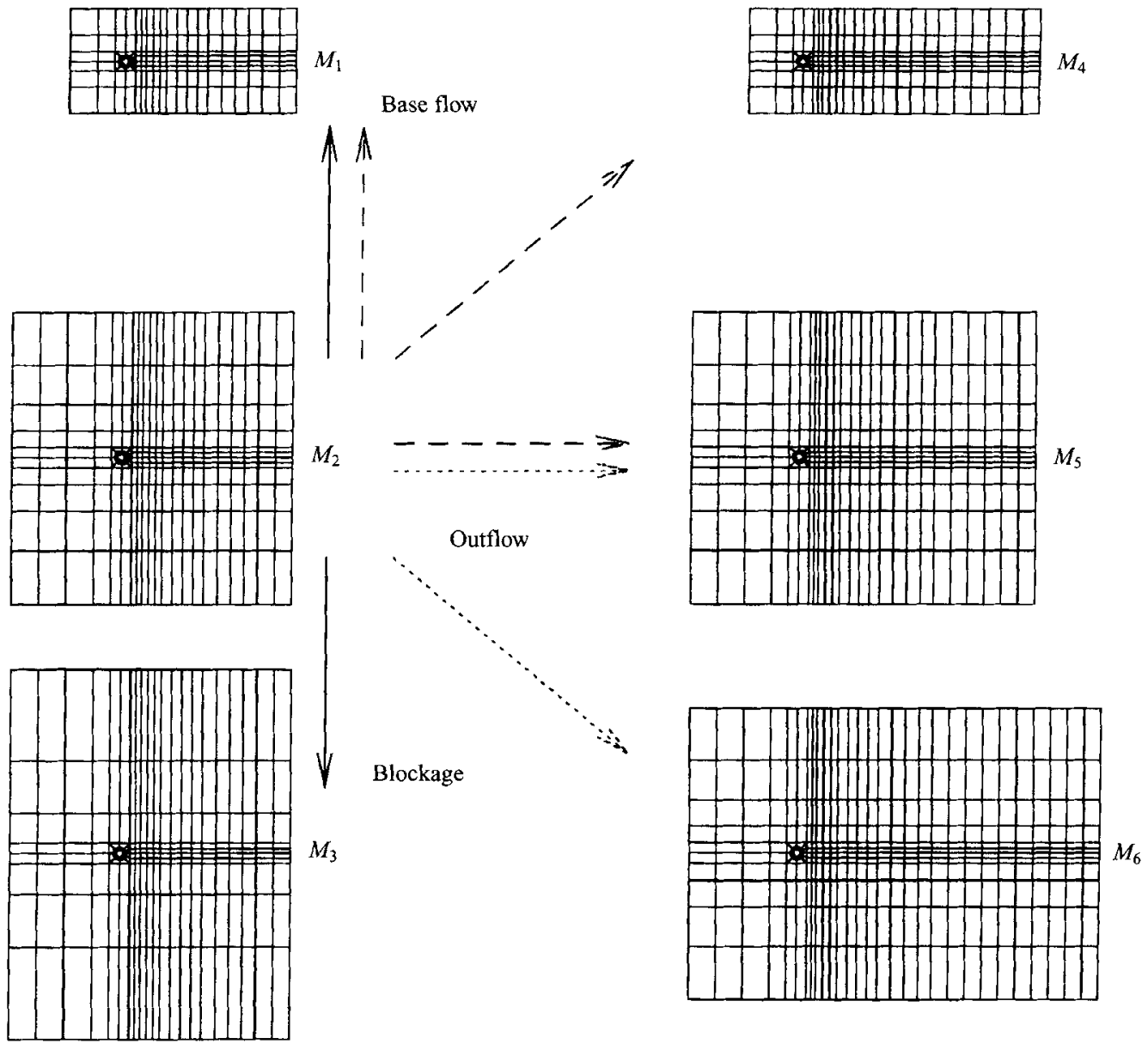

FiguRE 2. Family of spectral element meshes used for convergence studies. Arrows indicate schematically the comparisons made to test for blockage (solid arrows: $M_{1}-M_{2}-M_{3}$ ), outflow boundary effects (short-dashed arrows: $M_{2}-M_{5}-M_{6}$ ), and base flow dependence (long-dashed arrows: $M_{1}-M_{2}-M_{4}-M_{5}$ ). Each element shown contains $N^{2} \sim 16$ to 100 mesh points.

$$
\begin{array}{rrrrrrr} 
& M_{1} & M_{2} & M_{3} & M_{4} & M_{5} & M_{6} \\
K & 170 & 266 & 224 & 202 & 314 & 362 \\
l_{i} & 8 & 16 & 16 & 8 & 16 & 16 \\
l_{h} & 8 & 22 & 28 & 8 & 22 & 22 \\
l_{o} & 25 & 25 & 25 & 35 & 35 & 45
\end{array}
$$

TABLE 1. Defining parameters for the sequence of meshes $M_{1}-M_{6}$ used to check the influence of domain size and resolution on the base flow and stability calculations; $K$ is the number of elements; $l_{i}, l_{h}$, and $l_{l}$ are the inflow, crossflow, and outflow domain size parameters. 


$\begin{array}{ccccccc}N & \Delta t & S t & -C_{p b} & C_{D} & C_{D}^{\prime} & C_{L}^{\prime} \\ 4 & 1 \times 10^{-2} & 0.1936 & 0.9705 & 1.3922 & 0.0300 & 0.4839 \\ 6 & 1 \times 10^{-2} & 0.1955 & 0.9267 & 1.3389 & 0.0288 & 0.4601 \\ 8 & 1 \times 10^{-2} & 0.1954 & 0.9326 & 1.3442 & 0.0293 & 0.4655 \\ 10 & 5 \times 10^{-3} & 0.1954 & 0.9326 & 1.3442 & 0.0293 & 0.4656\end{array}$

TABLE 2. Convergence of global quantities with polynomial order $N$ on mesh $M_{3}$ at $\operatorname{Re}=190$.

(outflow), and $l_{h}$ (crossflow). Table 1 lists the number of elements and domain size parameters for each mesh. After establishing some basic trends with an initial pass through the entire range of Reynolds number, we performed a detailed study of the flow at $R e=190$. The study consisted of the following three steps.

First, we monitored convergence of the base flow with respect to polynomial order $N$ on a fixed computational mesh: $M_{3}$. This was done by computing values of the following global quantities: Strouhal number $S t$, base pressure coefficient $C_{p b}$, mean and fluctuating drag coefficients, $C_{D}$ and $C_{D}^{\prime}$, and fluctuating lift coefficient $C_{L}^{\prime}$. Our results for mesh $M_{3}$ are given in table 2, showing convergence to four digits for order $N \geqslant 8$. The convergence is dominated by convergence of the flow in the near wake, as this region is the most sensitive to spatial resolution. Since all our meshes are identical in the near-wake region, we concluded that at $R e=190$ a polynomial order of $N \geqslant 8$ was sufficient to resolve the flow in the near wake for any of our meshes.

Next, we checked the computational domain size (blockage and outflow boundary effects) by comparing the shedding frequency and vorticity distribution of converged solutions on the different meshes. Shedding frequency is a sensitive indicator of blockage effects; the shedding frequency increases with decreasing $l_{h}$ because of an effective upward shift in the local Reynolds number as the incompressible flow accelerates past the cylinder. We found no difference in shedding frequency for the meshes with $l_{h} \geqslant 22$ over the range of Reynolds number from 140 to 300 . The magnitude of vorticity at the centre of the wake vortices was used to check resolution of the flow downstream from the cylinder and to monitor the influence of the outflow boundary conditions on the flow upstream. Figure 3 shows the local extrema of vorticity for meshes of increasing outflow length, confirming that even the shortest mesh $M_{2}$ produces the correct wake to within 5 cylinder diameters of the outflow boundary.

Finally, we compared our simulation results with established experimental values over the entire range of Reynolds number. The comparison of Strouhal number is shown in figure 1 . In figure 4 we show a comparison of the base pressure coefficient, defined as $C_{p b} \equiv\left(p_{b}-p_{\infty}\right) / \frac{1}{2} \rho U_{\infty}^{2}$, where $p_{b}$ is the time-averaged pressure at the base $\left(180^{\circ}\right.$ from the front) of the cylinder and $p_{\infty}$ is the static pressure at infinity. $C_{p b}$ provides a sensitive characterization of drag on a bluff body. Figures 1 and 4 show clearly that our two-dimensional base flow calculations accurately reproduce the experimental measurements up to the point of transition.

For the stability computations we first address convergence of the eigenvalues under our iterative method for a fixed set of mesh parameters. At each iteration we compute the residual:

$$
r^{(n)}=\left\|\mathbf{A}\left(\tilde{\boldsymbol{u}}^{(n)}\right)-\mu^{(n)} \tilde{\boldsymbol{u}}^{(n)}\right\|
$$




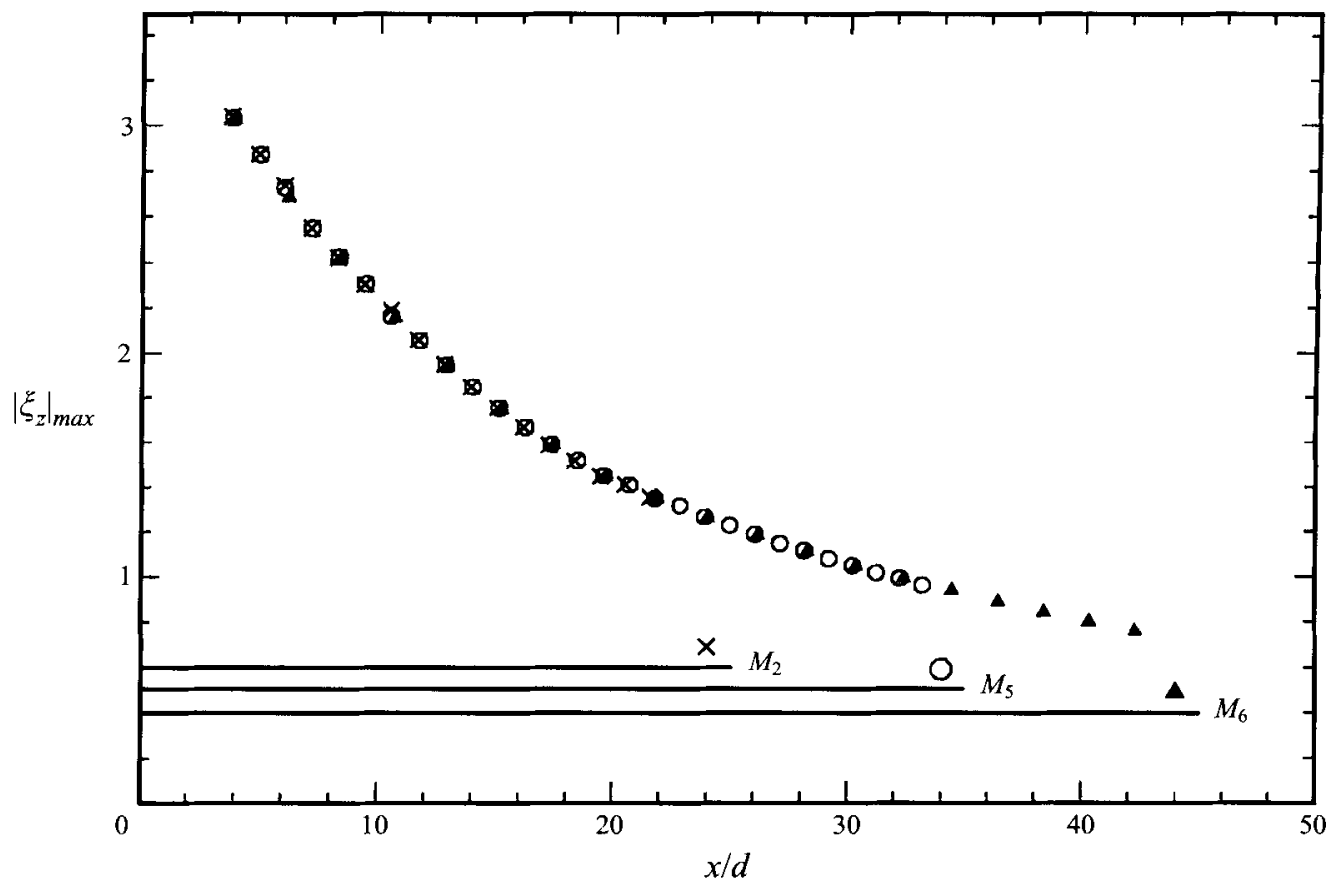

FIGURE 3. Variation of the local maximum in vorticity magnitude as a function of distance downstream from the cylinder for meshes $M_{2}, M_{5}$, and $M_{6}$ with symbols as indicated. Horizontal lines show the distance from the centre of the cylinder to the outflow boundary on each mesh.

$\begin{array}{ccc}\text { Iteration } & \mu & r \\ 7 & 1.00660 & 7.04 \times 10^{-2} \\ 8 & 1.01014 & 8.88 \times 10^{-3} \\ 9 & 1.01035 & 9.12 \times 10^{-4} \\ 10 & 1.01049 & 3.20 \times 10^{-4} \\ 11 & 1.01049 & 1.15 \times 10^{-5}\end{array}$

TABLE 3. Convergence of the dominant eigenvalue $\mu$ on mesh $M_{5}$ with $R e=190, \beta=1.6$.

where $\mu^{(n)}$ and $\tilde{\boldsymbol{u}}^{(n)}$ are the approximate eigenvalues and normalized eigenmodes $\left(\left\|\tilde{\boldsymbol{u}}^{(n)}\right\|=1\right)$ of $\mathbf{A}$ after the $n$th iteration, and $\|\cdot\|$ is the standard $L_{2}$ vector norm of the approximately $3 \times K \times N \times N$ values representing $\tilde{\boldsymbol{u}}$. As long as $r^{(n)}$ is small, $\mu^{(n)}$ will be an accurate eigenvalue of the operator A. Table 3 shows the convergence of eigenvalues as a function of iteration number for computations on the $M_{5}$ mesh with order $N=8$. The first iterations are not shown as they produce wholly inaccurate values of $\mu$.

In the eigenvalue computations we also tested for convergence with respect to polynomial order and sensitivity to domain size. The length scale of the eigenmodes is closely related to that of the corresponding base flow, and we found that they converged at roughly the same rate with respect to polynomial order. Again, order $N \geqslant 8$ gives well-converged results. Because the eigenmodes decay very rapidly away 


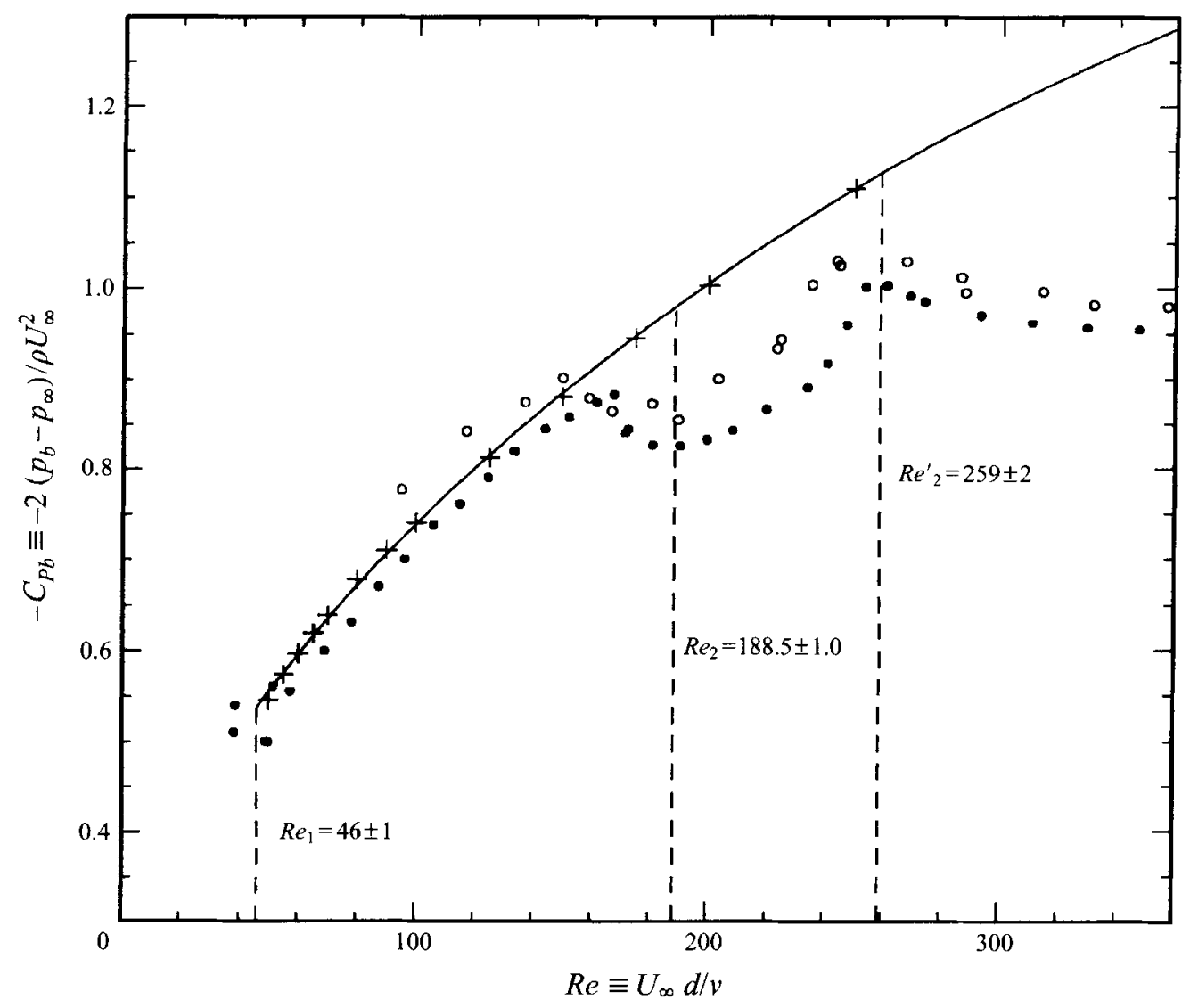

Figure 4. Variation of base pressure coefficient with Reynolds number from experiments and computations of two-dimensional flow: •, Williamson \& Roshko (1990); o, Norberg (1994); + , two-dimensional flow calculations performed as part of the current study (the solid line is a curve fit to the simulation data). The first few important wake instabilities are as labelled in figure 1. Note the sharp change in the slope of the base pressure curve at $R e_{2}^{\prime}$.

from the wake, the location of the external boundaries only has a minimal effect on the stability calculations. For example, we projected the converged base flow from the $M_{5}$ mesh at $R e=190$ onto each of the meshes $M_{1}, M_{2}$, and $M_{4}$. Because each of these meshes is an exact subset of the $M_{5}$ mesh, the resulting base flows are identical in the overlapping regions. The dominant eigenvalue computed for each base flow agrees with that for the $M_{5}$ case to 6 significant figures. Thus, the eigenvalue computations depend significantly on domain size only through the base flow and it is sufficient to perform the stability computations on the smallest mesh, $M_{1}$, using base flows computed on larger meshes.

Based on these tests, we chose the following protocol for our study near the critical Reynolds number for the secondary instability. Base flows are computed on mesh $M_{2}$ using order $N=8$ basis functions. These base flows are then projected onto mesh $M_{1}$, on which stability calculations are carried out also using polynomial order $N=8$. The eigenvalue iterations are halted when the residual falls below $10^{-3}$. We believe these results to be uniformly accurate to $0.2 \%$ in all global quantities. 


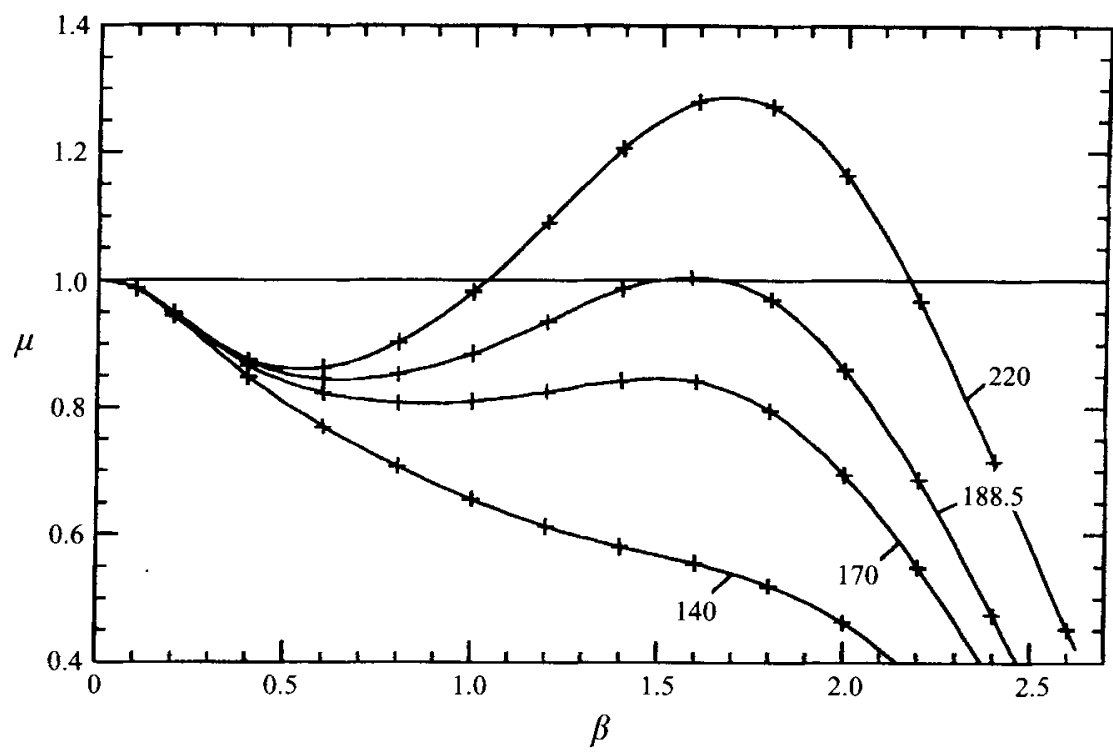

FIGURE 5. Dependence of the dominant Floquet multiplier $\mu$ on spanwise wavenumber $\beta$ for values of $R e$ indicated. The multipliers are real and positive. The value $\mu=1$ corresponds to the onset of instability. As $R e$ increases, the most unstable mode gradually shifts to a higher wavenumber (smaller wavelength). The cross size used to mark data points is chosen to indicate the accuracy of the multipliers (see text).

\section{Results}

In this section we show how the Floquet multipliers behave as a function of Reynolds number and spanwise wavenumber. Our main objective is to determine the precise values of $R e$ and wavenumber $\beta$ (or wavelength $\lambda=2 \pi / \beta$ ) for which the twodimensional wake is linearly unstable. Following this we present flow visualizations of the destabilizing Floquet modes that lead to three-dimensionality in the wake.

\subsection{Parameter dependence}

Figure 5 shows the dependence of the dominant Floquet multiplier (multiplier of greatest magnitude) on spanwise wavenumber $\beta$ for several values of the Reynolds number. Multiplier branches are symmetric with respect to a change of sign in $\beta$ and only $\beta \geqslant 0$ is plotted. The dominant multiplier is real and positive over the parameter range shown. Recall that the Floquet multiplier $\mu$ is related to the temporal exponent $\sigma$ via $\mu=\exp (\sigma T)$, where $T$ is the wake period, so that $|\mu|=1$ corresponds to zero exponential growth of linear modes.

Owing to time-translation symmetry there is a Floquet multiplier $\mu=1$ at $\beta=0$, regardless of the value of $R e$. This is because any autonomous time-periodic flow has a neutrally stable Floquet mode of the form $\tilde{\boldsymbol{u}}_{T} \propto \partial \boldsymbol{U} / \partial t$ (see e.g. Guckenheimer \& Holmes 1983). This mode 'points' along the periodic orbit. A two-dimensional mode corresponds to $\beta=0$, and $\tilde{\boldsymbol{u}}_{T}$ is two-dimensional because the base flow $\boldsymbol{U}$ is. We verified that the neutral Floquet modes obtained from our computations at $\beta=0$ are indeed of this form.

The behaviour of the multipliers away from $\beta=0$ depends on $R e$ as shown. For $R e=140$, the multiplier decreases monotonically as a function of $\beta$. At $R e=170$, the multiplier branch has a local maximum at finite $\beta$. This maximum grows and shifts to 


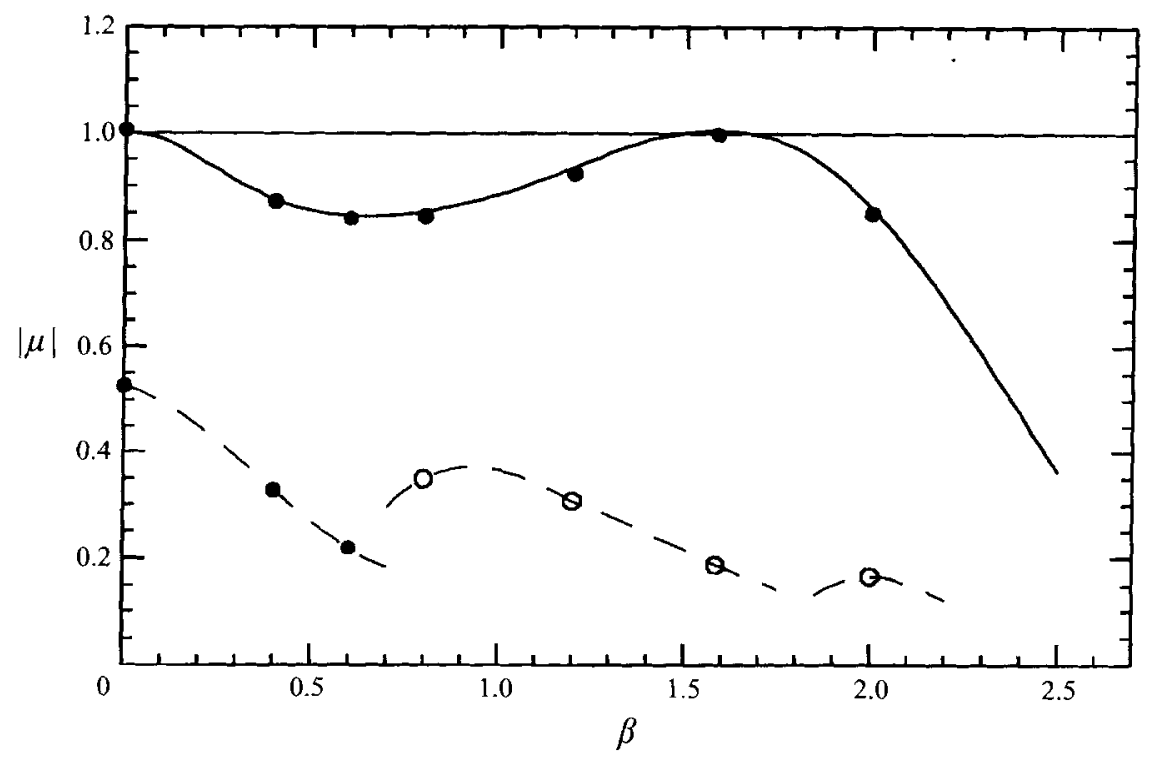

FIGURE 6. Floquet multiplier branches as a function of spanwise wavenumber $\beta$ at the critical Reynolds number $R e_{2}=188.5$. At each value of $\beta$, the two multipliers of greatest magnitude are plotted. Filled circles indicate real, positive multipliers; hollow circles indicate complex-conjugate pair multipliers. The solid curve is repeated from the figure 5. Dashed curves are not exact but give some indication of subdominant multiplier branches.

a slightly higher wavenumber as $R e$ is increased, reaching $\mu=1$ at the critical values: $R e_{2}=188.5, \beta_{2}=1.585$. Our determination of the precise critical values is discussed below. Note that the critical Floquet mode for the onset of secondary instability is on the same branch of multipliers as the neutral mode owing to time-translation symmetry. For $R e>R e_{2}$ there is a band of wavenumbers for which $\mu>1$. There are no further instabilities at higher $\beta$ for the Reynolds numbers shown in figure 5 . We return to the issue of high-wavenumber instabilities below.

The results shown in figure 5 are from parameter scans in which both the base flow and stability computations were run on the small $\left(M_{1}\right)$ mesh with polynomial order $N=6$. However, we performed fully resolved computations at selected parameter values to confirm that these results are accurate to within about $1 \%$. The cross size used to mark data points was chosen to indicate the accuracy of the multipliers. Except where noted, all further results presented are from fully resolved computations using the protocol described in $\$ 2.3$.

Figure 6 shows additional details of the Floquet multiplier spectrum at the secondary instability: $R e=R e_{2}$. The solid curve is repeated from the preliminary calculations shown in figure 5 . Note that the fully resolved computations here agree closely with this curve. Other multiplier branches are shown with dashed curves. We did not resolve the details of these multiplier branches and the dashed curves are only meant to give some indication of their behaviour. At any given value of $\beta$ there are no other multipliers between the branches shown and thus the dominant branch of Floquet multipliers is well separated from other multipliers for wavenumbers up to 2.5. We did not attempt to compute deeper into the multiplier spectrum, i.e. smaller $|\mu|$.

The wake is quite stable two-dimensionally at the secondary instability. This follows from the fact that at $\beta=0$ the first multiplier, other than that due to time-translation 


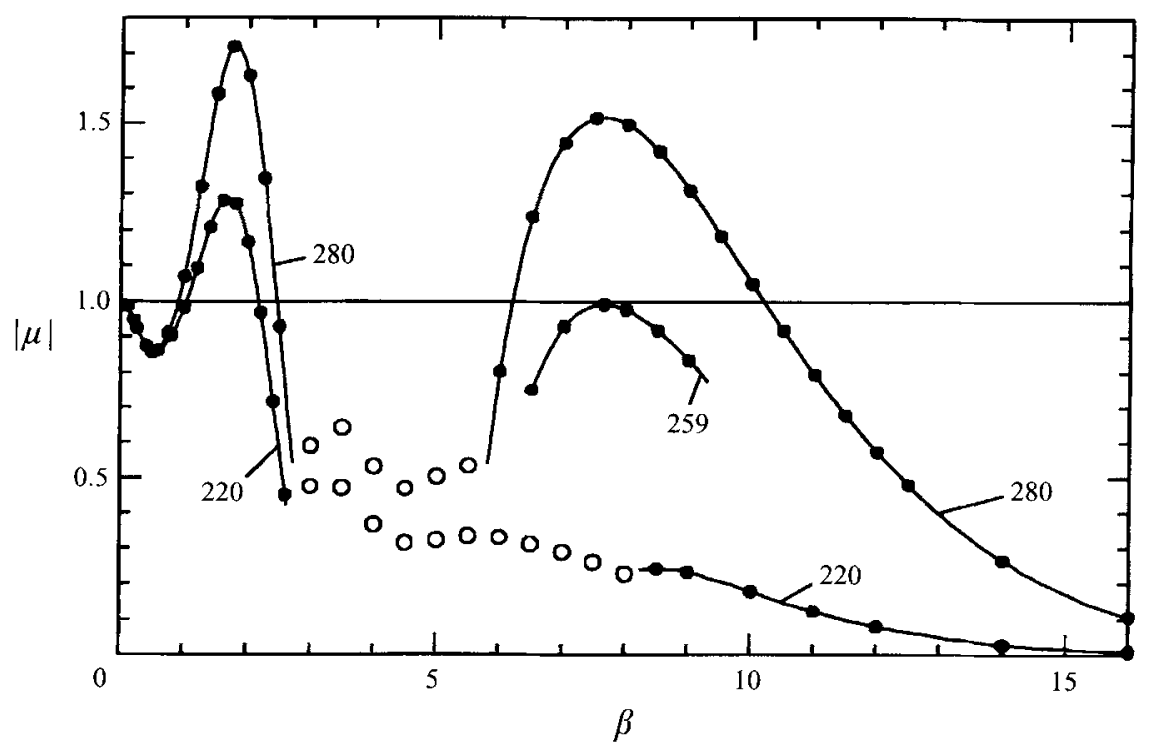

FIgure 7. Dominant Floquet multipliers as a function of spanwise wavenumber $\beta$ at Reynolds numbers $R e=220, R e=259$, and $R e=280$. For clarity, only a portion of the spectrum $(6.5 \leqslant \beta \leqslant 9.3)$ at $R e=259$ is plotted in the vicinity of the local maximum: $\mu=1, \beta=7.64$. Filled circles indicate real, positive multipliers; hollow circles indicate complex-conjugate pair multipliers. Branches of real multipliers at both low and high $\beta$ are connected by solid lines.

symmetry, has a value $\mu=0.52$ corresponding to a linear perturbation that decreases by a factor of about 2 each wake period. We did not attempt accurate computations of the second multiplier branch at $\beta=0$ for other values of $R e$, but in general we found that over the range of our computations $(R e \leqslant 300)$ the wake is two-dimensionally stable. The value $\mu=0.52$ is in agreement with the result of Noack \& Eckelmann (1994a) that $|\mu| \approx 0.6$ throughout the range $50<R e<300$. The two-dimensional stability of the cylinder wake over this range of Reynolds number can also be inferred from previous direct numerical simulations (e.g. Karniadakis \& Triantafyllou 1989, 1992).

Figure 7 shows the behaviour of the dominant Floquet multiplier over a wider range of spanwise wavenumbers. It can be seen that at $R e=220$ the multipliers remain small in magnitude for all $\beta$ larger than about 2.5 , and hence the only unstable wavenumbers for $R e=220$ and lower $R e$ are those shown in figure 5 . In the range $3 \lesssim \beta \lesssim 8$ the dominant multipliers are complex and we did not attempt to resolve the details of these multiplier branches. For $\beta \geq 8$ the dominant multiplier is again real and positive. We verified that as $\beta$ becomes large $(\beta>12)$ this multiplier goes to zero as $\ln \mu \sim-\beta^{2}$. This scaling is to be expected because the viscous term $\left(\partial^{2} / \partial z^{2} \equiv-\beta^{2}\right)$ begins to dominate the linearized equations when $\beta \sim R e^{1 / 2}$. Once this viscous-dominated regime is reached, no further instabilities are possible for larger $\beta$.

As the Reynolds number is increased above 220 , the maximum in the real multiplier branch at $\beta \approx 8$ grows, reaching $\mu=1$ at $R e_{2}^{\prime}=259 \pm 2$, as shown in figure 7 . The associated wavenumber is $\beta_{2}^{\prime}=7.64 \pm 0.06$ corresponding to a spanwise wavelength of $\lambda_{2}^{\prime}=0.822 \pm 0.007$ cylinder diameters. For larger $R e$ there are two bands of unstable wavenumbers, as shown for the case $R e=280$. 


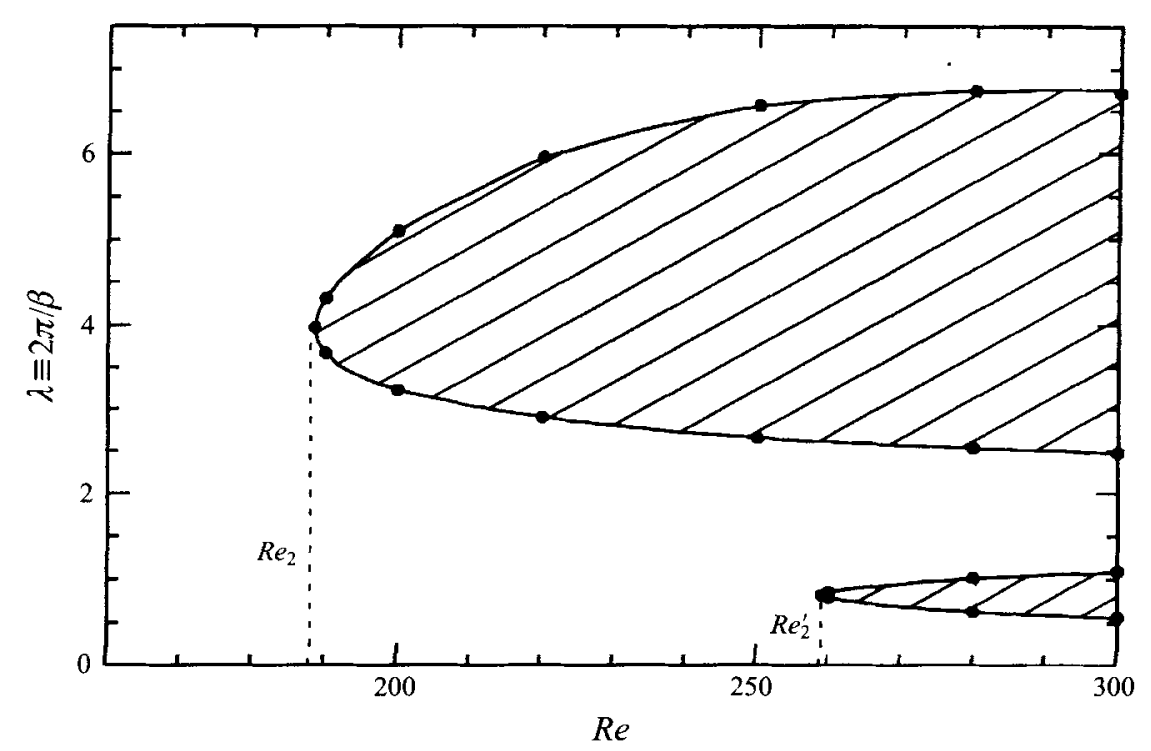

Figure 8. Curves of neutral stability for the cylinder wake. Everywhere in the shaded regions there exist exponentially growing three-dimensional modes of the two-dimensional wake. The critical Reynolds number for the long-wavelength region is $R e_{2} \simeq 188.5$. The short-wavelength region is a separate instability with a critical Reynolds number of $R e_{2}^{\prime} \simeq 259$.

The multiplier branch at $R e=259$ was determined by fully resolved computations. However, as with figure 5, the spectra shown in figure 7 at $R e=220$ and $R e=280$ are from computations in which both the base flow and stability computations were run on the small $\left(M_{1}\right)$ mesh with polynomial order $N=6$.

Figure 8 shows two curves of neutral stability for the wake. By convention we plot spanwise wavelength $\lambda=2 \pi / \beta$ as a function of Reynolds number. Everywhere along a neutral curve there exists a multiplier $\mu=1$ and to the right of these curves the cylinder wake is linearly unstable to three-dimensional perturbations. Points along the neutral curves were found by computing multipliers at parameter values in the vicinity of $\mu \approx 1$, and then interpolating to find $\mu=1$. The curves plotted in figure 8 are polynomial fits through these points.

The critical values $R e_{2}$ and $\lambda_{2}=2 \pi / \beta_{2}$ for the onset of three-dimensionality were determined by two methods. First, stability computations were performed at $R e=187$ and at $R e=190$ for $\beta=1.4,1.5,1.6$, and 1.7. From these data a two-dimensional fit (first order in $R e$ and third order in $\beta$ ) gives critical values: $R e=188.6, \lambda=3.967$ (or $\beta=1.584$ ). As a second, largely independent determination of the critical values, the four points on the neutral curve at $R e=190$ and $R e=200$ (figure 8) were fit with a second-order polynomial and from this fit the critical point of the neutral curve was found to be: $\operatorname{Re}=188.5, \lambda=3.954(\beta=1.589)$. The two methods are not entirely independent, however, because both use some of the same data at $R e=190$. We took the critical values to be: $R e_{2}=188.5 \pm 1.0$ and $\lambda_{2}=3.96 \pm 0.02\left(\beta_{2}=1.585 \pm 0.010\right)$, where the error bounds are conservative and based on our overall estimation of the errors in the flow fields and stability computations and how these propagate in the interpolations used to find critical values. Stability computations at $R e=188.5$, $\beta=1.585$ give $\mu=0.9972$.

The minimum Reynolds number and associated wavelength for the crossing of the high-wavenumber branch of multipliers was determined similarly, though we did not 
strive for as great a precision in this case. From stability computations at $R e=257$ and $R e=260$ we find $R e_{2}^{\prime}=259 \pm 2$ and $\lambda_{2}^{\prime}=0.822 \pm 0.007\left(\beta_{2}^{\prime}=7.64 \pm 0.06\right)$, where again the error bounds are quite conservative. Stability computations at $R e=259$, $\beta=7.64$ using the protocol described in $\$ 2.3$ give $\mu=0.992$. As a final check we repeated the computations at $\operatorname{Re}=259, \beta=7.64$ with higher spatial resolution $(N=10)$ and obtained $\mu=0.995$.

\subsection{Critical Floquet modes}

We turn now to a description of the critical Floquet modes driving the threedimensional instability in the cylinder wake. First, recall from equation (2.6) that the Floquet modes have a simple $z$-dependence:

$$
\tilde{\boldsymbol{u}}(x, y, z, t)=(\hat{u} \cos \beta z, \hat{v} \cos \beta z, \hat{w} \sin \beta z),
$$

from which the form of their vorticity follows:

$$
\tilde{\xi}(x, y, z, t)=\left(\hat{\xi}_{x} \sin \beta z, \hat{\xi}_{y} \sin \beta z, \hat{\xi}_{z} \cos \beta z\right)
$$

The origin of $z$ here is arbitrary and any translation of (3.1) or (3.2) along the span is also a Floquet mode.

To visualize the instability, we consider a superposition of the two-dimensional wake $U$ and the critical Floquet mode $\tilde{\boldsymbol{u}}$ at $R e=R_{2}, \beta=\beta_{2}$. This superposition takes the form $U+\tilde{\boldsymbol{u}}$, and should mimic the three-dimensional flow close to the onset of the instability. In the norm discussed earlier, $\|\tilde{\boldsymbol{u}}\| \simeq 0.01\|\boldsymbol{U}\|$ so that the superposition represents about a $1 \%$ perturbation to the base flow. Figure 9 shows the resulting three-dimensional wake at one instant in time, visualized as surfaces of constant streamwise vorticity and spanwise vorticity magnitude. The spanwise vorticity is dominated by the primary von Kármán vortices, the effect of the Floquet mode being a spanwise-periodic perturbation to these vortices at the critical wavelength $\lambda_{2}=2 \pi / \beta_{2} \simeq 4$ diameters. Linking the von Kármán vortices are thin patches of streamwise vorticity due entirely to the destabilizing Floquet mode. From equations (3.1) and (3.2) it follows that the streamwise and spanwise vorticity are out of phase in the $z$-direction, i.e. points of zero streamwise vorticity are aligned with points of maximum deformation in the von Kármán vortices and vice versa. As discussed in the next section, this three-dimensional flow strongly resembles what Williamson (1988) refers to as 'Mode A' vortex shedding.

While figure 9 clearly illustrates the full three-dimensional form of the instability, many details can be better seen in figure 10 where the streamwise vorticity $\hat{\xi}_{x}(x, y, t)$ and spanwise vorticity $\hat{\xi}_{z}(x, y, t)$ are plotted over one half-period of the Floquet mode. Spanwise vorticity in the growing mode is an order of magnitude stronger than streamwise vorticity. An important observation from figure 10 is that streamwise vorticity is generated close to the cylinder and rapidly injected into the wake. It originates as a vorticity dipole in the region between the separating shear layers and moves outward, emerging at the edge of the wake around $x / d \simeq 2$. The thin patches of streamwise vorticity that develop do not persist more than a few diameters downstream in the Floquet mode. Also, the dipole component farthest from the centreline rapidly decays so that by the time it reaches $x / d \simeq 4$ only a single sign of streamwise vorticity remains at a given spanwise location. When superimposed on the two-dimensional base flow, a pair of counter-rotating streamwise vortices would appear to emerge from the near wake, while the dipole structure would remain largely hidden inside the recirculation zone.

It can be seen that the spanwise vorticity dipole aligns roughly with the centre of 


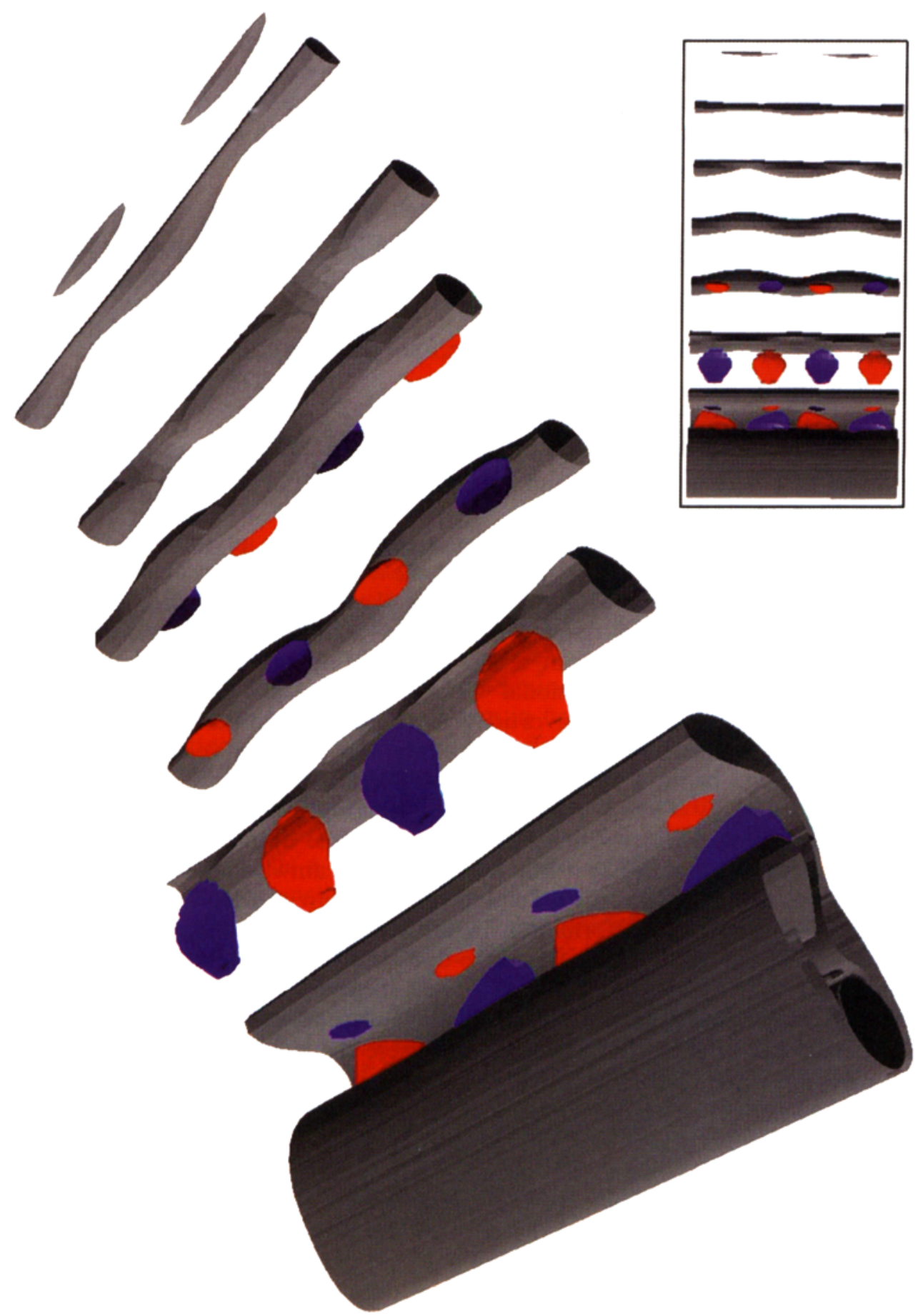

Figure 9. Superposition of the critical Floquet mode on the von Kármán vortex street. The grey tubes are isocontours of spanwise vorticity magnitude, $\left|\xi_{z}+\hat{\xi}_{z}\right|$. The critical Floquet mode gives rise to a spanwise-periodic deformation of the primary von Kármán vortices. Linking these vortices are thin regions of positive (red) and negative (blue) streamwise vorticity, $\hat{\xi}_{x}$. The inset shows a view of the same structure from above. Data for this figure were taken from image $(a)$ of figure 10. 
Stability analysis of the cylinder wake

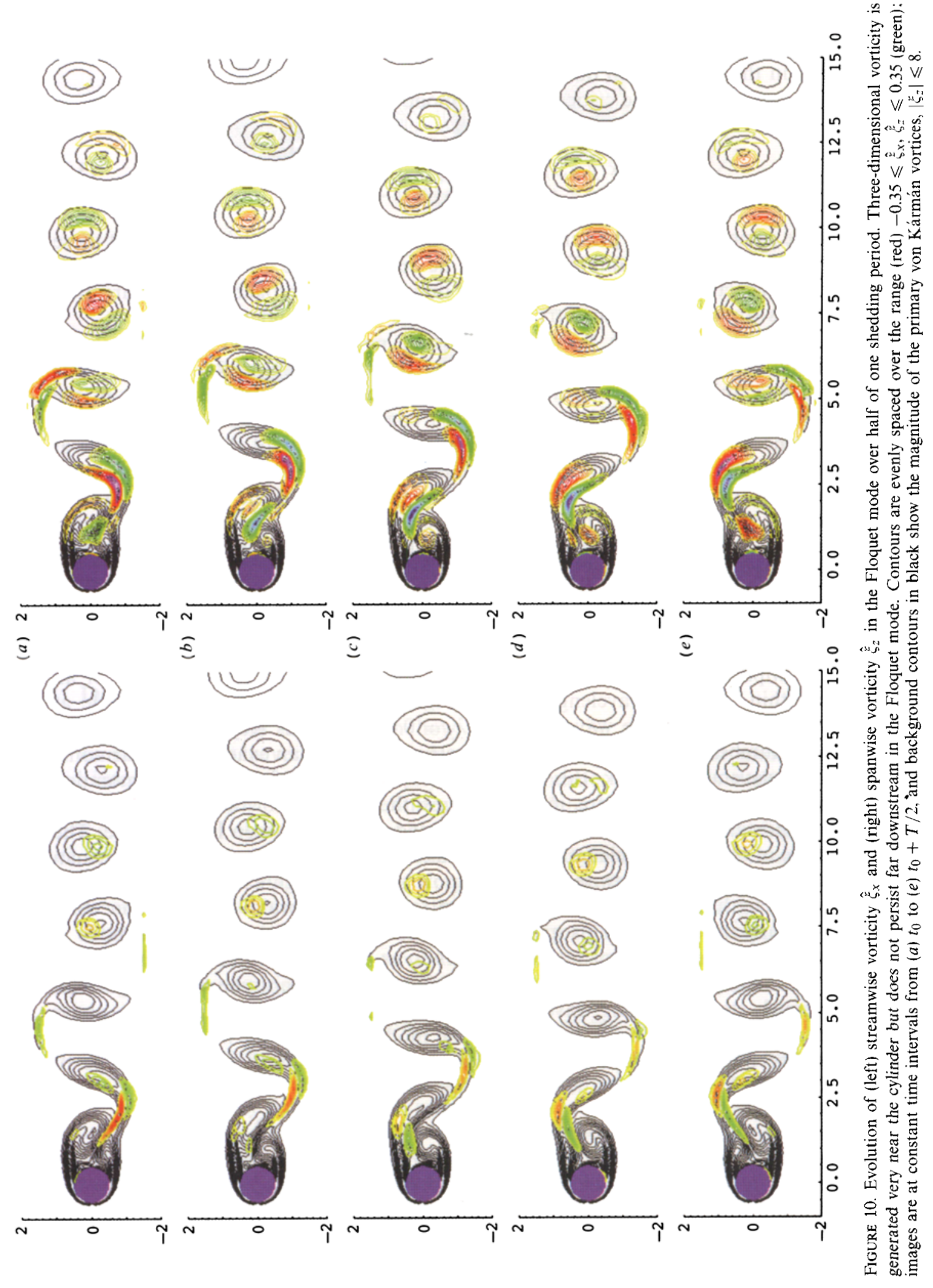


each von Kármán vortex, but that the downstream component is slightly stronger. To the extent that the flow after transition is well-approximated by our superposition, this means that vortex lines associated with each von Kármán vortex will be displaced primarily in the downstream direction.

Finally, we note that the critical Floquet mode has the spatio-temporal symmetry

$$
\left.\begin{array}{rl}
\tilde{u}(x, y, z, t) & =\tilde{u}(x,-y, z, t+T / 2), \\
\tilde{v}(x, y, z, t) & =-\tilde{v}(x,-y, z, t+T / 2), \\
\tilde{w}(x, y, z, t) & =\tilde{w}(x,-y, z, t+T / 2) .
\end{array}\right\}
$$

This is the same symmetry present in the two-dimensional wake (with $w=0$ ) at these values of the Reynolds number. This can be seen in figure 10 where image $(e)$ is simply a $y$-reflection of image $(a)$. Thus, the only symmetry broken at the bifurcation point $R e_{2}$ is translational symmetry along the axis of the cylinder.

We now consider briefly the critical Floquet mode associated with the shortspanwise-wavelength instability originating at the critical point $R e_{2}^{\prime}$. For reasons given in $\$ 4.2$, this mode is unstable at onset and so we do not describe it in the same detail as the long-wavelength mode just considered. Instead we focus just on the differences between the two modes. Although the vorticity of the critical mode at $R e_{2}^{\prime}$ has the same general dipole form as the critical mode at $R e_{2}$, there are important differences in the structure and spatio-temporal symmetries of the two modes. In the next section we also discuss how these differences relate to the physical instability mechanisms described by Williamson (1996c), but here we limit ourselves to what may be observed directly from the linear stability calculations.

Figure 11 shows a qualitative comparison of the two modes with the largest linear growth rate within each unstable wavelength band at $R e=259$. The mode in figure $11(a)$ is the (unstable) long-wavelength mode corresponding to a maximum in the multiplier spectrum at $\lambda=3.28 \mathrm{~d}$. This mode is a good representative of all modes within the long wavelength band. Figure $11(b)$ shows the critical mode at $R e_{2}^{\prime}$, which has a spanwise wavelength of $\lambda_{2}^{\prime}=0.82 d$. At this Reynolds number the high-wavenumber peak in the multiplier spectrum is almost an exact fourth harmonic of the low-wavenumber peak; this is not true at other values of the Reynolds number. Figure 11 allows us to compare, in broad terms, the character of the two instabilities.

One important difference between the two modes is clearly seen in the figure. The magnitude of the long-wavelength mode is peaked around the core of the von Karman vortices, just as is the spanwise vorticity dipole shown in figure 10 . In contrast, the short-wavelength mode is much more concentrated in the 'braid' regions connecting the von Kármán vortices. This general structure for the short-wavelength mode is consistent with what Williamson (1988) refers to as 'Mode B' vortex shedding.

The second important difference between the two modes cannot be seen in figure 11 . As previously stated, the long-wavelength Floquet mode at the critical point $R e_{2}$ obeys the symmetry in (3.3). This is true for all the modes on the long-wavelength (shortwavenumber) branch. The critical mode at $R e_{2}^{\prime}$ corresponds to a different type of symmetry breaking. It obeys the spatio-temporal symmetry

$$
\left.\begin{array}{rl}
\tilde{u}(x, y, z, t) & =\tilde{u}\left(x,-y, z+\lambda_{2}^{\prime} / 2, t+T / 2\right), \\
\tilde{v}(x, y, z, t) & =-\tilde{v}\left(x,-y, z+\lambda_{2}^{\prime} / 2, t+T / 2\right), \\
\tilde{w}(x, y, z, t) & =\tilde{w}\left(x,-y, z+\lambda_{2}^{\prime} / 2, t+T / 2\right) .
\end{array}\right\}
$$

This is also a symmetry of the base flow. All modes on the short-wavelength (high-wavenumber) branch have this symmetry. 


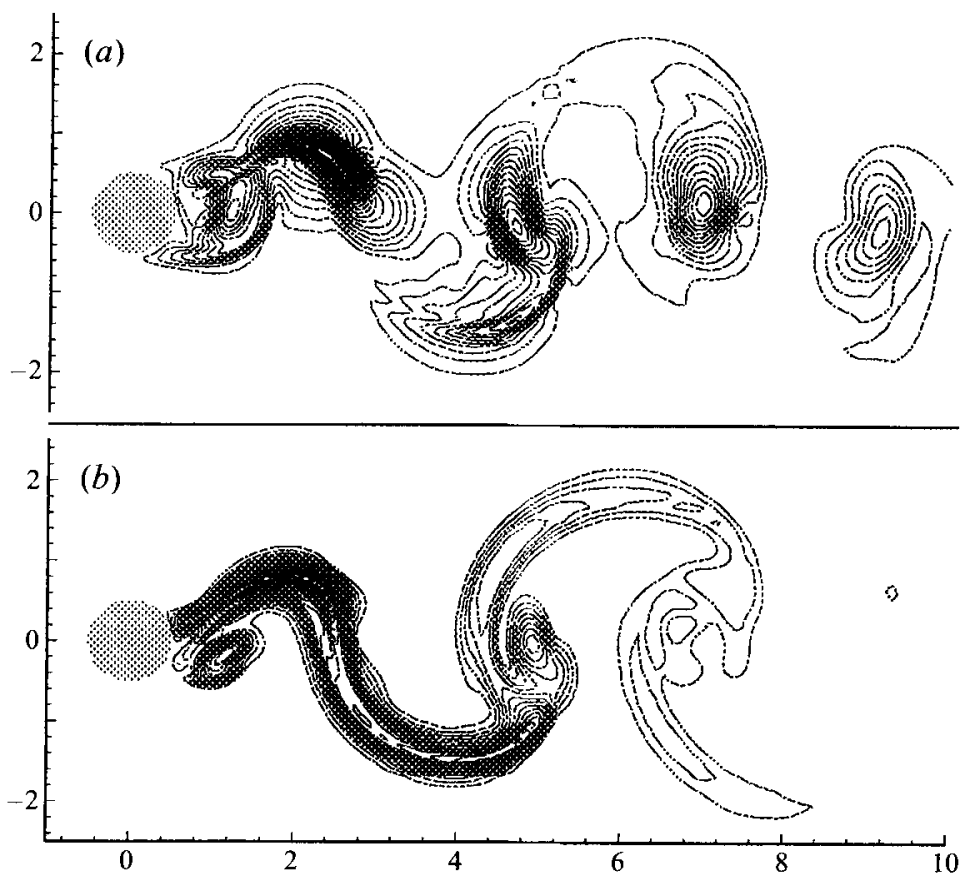

FIGURE 11. Qualitative comparison of the structure of the most unstable long- and short-wavelength modes at $R e_{2}^{\prime} \simeq 259$. Contours of $|\tilde{u}|$ are shown for the Floquet modes with spanwise wavelength (a) $\lambda=3.28 d$ and $(b) \lambda=\lambda_{2}^{\prime}=0.82 d$. Although the base flow vorticity is not shown, $(a)$ is largely concentrated in the spanwise vortex cores while $(b)$ is stronger in the braid regions connecting the vortex cores.

\section{Discussion}

While linear theory is limited in how far it may be applied beyond the onset of an instability, it nevertheless provides invaluable information for understanding and interpreting observations from physical experiments. Quantities such as critical parameter values (e.g. Reynolds number and wavelength) can be compared directly with experimental observations, and the most dangerous linear modes often capture the essential structure of the flow observed in the nonlinear physical system. In the present case, we find an excellent correspondence between the predictions of our linear stability calculations and recent experimental work over a fairly wide range of Reynolds number above $R_{2}$. Although precise quantitative comparison with experiment is difficult for reasons we outline below, the calculations and experiments together present a consistent picture of secondary instability in the wake.

\subsection{Critical Reynolds number}

From our linear stability analysis we determined the critical Reynolds number for the secondary instability of the two-dimensional von Kármán vortex street to be $R e_{2}=188.5 \pm 1.0$. By definition, this is the minimum value of the Reynolds number such that the Floquet spectrum includes a neutrally stable mode $(|\mu|=1)$ with a non-zero wavenumber. If perturbed by an infinitesimal amount, this mode neither grows nor decays. A direct comparison with experimental estimates of the critical Reynolds number is complicated by important factors that require some elaboration.

Most important is the degree to which a flow created in the laboratory approximates the ideal conditions of flow past an infinitely long cylinder. It has been found that 
the experimental techniques used to control end effects have an important influence on the wake dynamics even for seemingly large-aspect-ratio systems. Miller \& Williamson (1994) and Williamson (1996b) suggest that contamination from end effects is responsible for the large scatter in values of the critical Reynolds number observed in previous experimental work (140 to 190). In comparing with experiment, it is appropriate to consider only studies where end effects are well-controlled and the wake remains relatively 'clean' up to the point of transition.

The second complication is related to the nonlinear form of the bifurcation. In a separate publication (Henderson \& Barkley 1996), we show that the secondary instability is weakly subcritical: there is a small range of bistability between the twodimensional flow and the three-dimensional flow bifurcating from $R e_{2}$. This bistability results in a hysteresis during the transition between the two states near $R e_{2}$, depending on whether the control parameter $(R e)$ is increased or decreased. Hysteresis is shown clearly in the experimental data of Williamson (1988) and Leweke \& Provansal (1995). Strictly speaking, the critical point is physically unreachable because it is absolutely unstable to finite-amplitude perturbations. Even low-amplitude disturbances, always present in experiments, can trigger three-dimensional transition at $R e<R e_{2}$ and produce an apparent scatter in the critical Reynolds number that corresponds to the onset of the linear instability. Although this hysteresis effect is much more delicate than potentially large-scale effects due to end conditions, it is a consideration in interpreting experimental results for Reynolds numbers in the range 180 to 190 .

Another consideration in comparing experiments to our linear stability calculations is the technique commonly used to estimate the 'critical' Reynolds number in experiments. The appearance of three-dimensionality in the physical wake is associated with a significant drop in shedding frequency (see figure 1). The point where this drop occurs is generally reported as the critical or, more appropriately, the transition Reynolds number. However, at the secondary instability the leading Floquet multiplier is real and positive and as a result no new temporal frequency is introduced. Based on our computations, we conclude that this is not a linear effect of the instability, but is brought about by strongly nonlinear phenomena observed at $R e \simeq R e_{2}$ and above. These include, most notably, the occurrence of 'vortex dislocations' in the near wake as discussed by Williamson (1992). Other complex changes in the Strouhal-Reynolds number relationship are discussed by Leweke \& Provansal (1995), Zhang et al. (1995), and particularly in the context of the critical Reynolds number by Williamson $(1996 a)$.

With these points in mind, we note the following recent experimental estimates of $R e_{2}$. In an investigation of oblique and parallel vortex shedding with low turbulence levels and careful control of end effects, Williamson (1989) finds the onset of threedimensionality in the wake at $R e \approx 180$. In more recent work, Miller \& Williamson (1994) use 'end suction' to control spanwise contamination due to end effects and extend the 'laminar' shedding regime to $R e \approx 194$. Leweke \& Provansal (1995) report measurements for the wake of a ring, which for large aspect ratios behaves much like a straight cylinder but without end effects. For such configurations they find a drop in shedding frequency at $R e \approx 185$, and report an upper stability limit for laminar periodic shedding that never exceeds $R e \approx 190$ for a variety of ring aspect ratios. In spite of the difficulties outlined above, we feel these results are in excellent agreement with the critical Reynolds number of $R e_{2} \simeq 188.5$ determined from our linear stability calculations.

We wish to re-emphasize that, owing to the various effects elaborated above, one cannot precisely determine the critical point $R e_{2}$ of the linear instability either by 
observing the onset of three-dimensionality in flow visualization or by measuring changes in the shedding frequency. To estimate the critical point accurately, one should instead measure the decay rate of disturbances at parameter values prior to the onset of the instability and extrapolate to find the parameter value corresponding to zero decay rate (see e.g. Schatz et al. 1995). In effect, this is the procedure for measuring eigenvalues (decay rates) in the physical system and is analogous to the numerical method for analysing the leading behaviour in the eigenvalue problem.

Finally we note that Noack \& Eckelmann $(1994 a, b)$ find $R e_{2} \simeq 170$ from a numerical Floquet stability analysis. While this value is about $10 \%$ below the actual value, they are able to compute this result by examining the stability of a low-dimensional dynamical system obtained by Galerkin projection of an analytical basis composed of relatively few (order $10^{2}$ ) spatial modes. In comparison, our calculations involve order $10^{4}$ spatial degrees of freedom. Zhang et al. (1995), in both finite difference calculations and experiments in a water tunnel, are able to induce (using a small wire in the near wake) a three-dimensional flow at $R e \approx 170$ with a wavelength similar to that found in the low-dimensional model. They suggest that somehow the low resolution of the model indicates the instability of this particular mode. Based on our findings, we can only say that such a mode would be stable in the natural wake.

\subsection{Spatial structure of the three-dimensional flow}

Williamson (1988) identifies two modes of three-dimensional vortex shedding after transition, characterized by the streamwise vorticity inferred from laser-induced fluorescent dye visualization: a relatively long-wavelength, regular pattern ('Mode A') for Reynolds numbers in the range $180<R e<230$, and a finer-scale, irregular pattern ('Mode B') that emerges for $R e$ between 230 and 260 ; above $R e \approx 260$ the finer-scale mode appears to dominate. The development of each mode is associated with a distinct change in measurable quantities such as the shedding frequency, discussed above, and the base pressure coefficient (see figure 4). Leweke \& Provansal (1995) note additional changes in measured spanwise correlations at $R e \approx 180$ and $R e \approx 260$. The value of Reynolds number where these changes occur is naturally associated with the two critical points, $R e_{2}$ and $R e_{2}^{\prime}$, of the neutral stability curves shown in figure 8 . The neutral stability curves also provide a framework for discussing the development of the three-dimensional flow above the onset of the secondary instability and are reproduced in figure 12, along with experimental wavelength measurements over the same range of Reynolds number.

A qualitative comparison is possible between the three-dimensional structure of the flow that bifurcates from $\mathrm{Re}_{2}$ and 'Mode A' observed in experiments. As stated above, the secondary instability is only weakly subcritical. Therefore, the linear approximation obtained by superimposing the base flow and critical Floquet mode is reasonably faithful to the saturated nonlinear state. Of course, this approximation will be best for $R e \approx R e_{2}$ and at short times during the transition process. Experimental results presented by Williamson (1992, figure 2) represent the ideal case for comparison - images taken from water towing-tank runs near the critical point $R e_{2}$ just as threedimensionality first appears. Indeed, the flow obtained in this way exhibits the same spatial structure as the critical Floquet mode presented in figure 9.

A more quantitative comparison between the shedding modes and our stability computations can be made in terms of the spanwise wavelengths observed in experiments. Since most experimental observations are made under steady operating conditions, these wavelengths reflect the final three-dimensional state after transition and not the instability itself. Again, the best comparison between our calculations 


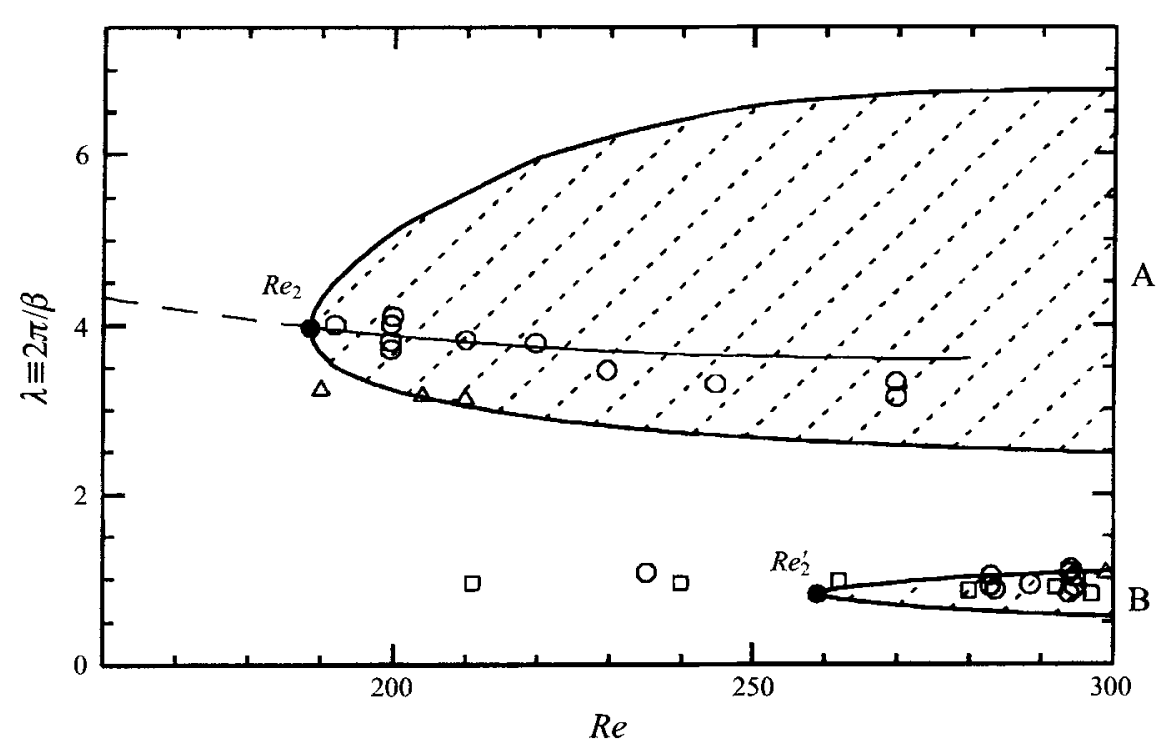

FIgURE 12. Comparison of the regions containing linearly unstable modes with the dominant spanwise wavelength observed in experiments: $\square$, Wu et al. (1994); $\triangle$, Mansy et al. (1994); o, Williamson (1996a); $\bullet$, critical points from our linear stability calculations. The solid line gives an indication of the wavelength with the largest linear growth rate up to $R e=280$. With the exception of Williamson (1996a), these measurements are taken in the near wake under steady operating conditions and do not correspond to the onset of the instability.

and experiments near the critical point $R e_{2}$ can be made in cases where threedimensionality first starts to appear. Wavelengths measured this way by Williamson (1996a) fall close to the most unstable modes shown in figure 12, quoted as $\lambda=4.01$ diameters at $R e=192$, in excellent agreement with our computations. It would seem that linear stability theory gives a rather complete description of the bifurcation corresponding to 'Mode A' in the wake.

Comparison in the case of 'Mode B' is more problematic. The critical Floquet mode at $R e_{2}^{\prime}$ is localized to the near wake, decaying quickly to zero beyond $x \simeq 6$ diameters. Near the cylinder, a linear superposition of this mode and the twodimensional wake does resemble 'Mode B', both in terms of qualitative structure and the symmetry of streamwise vorticity reported by Williamson (1996b). Still, a detailed comparison between experiments and linear stability calculations in this case is questionable for the following reason. The three-dimensional flow that bifurcates from the two-dimensional wake at $R e_{2}^{\prime}$ is necessarily unstable whether the bifurcation is subcritical or supercritical. This is because the two-dimensional wake is already unstable to 'Mode $A$ ' at this Reynolds number and the three-dimensional branch that bifurcates inherits this instability. Any bifurcation from $R e_{2}^{\prime}$ will not reach an equilibrium consisting purely of 'Mode B' because it is also unstable to longerwavelength perturbations. Even if 'Mode B' is dominant, the physical wake could consist of some mix of modes from both unstable regions over the range of Reynolds numbers contained in figure 12.

Clearly the flow arising from the bifurcation to 'Mode B' involves nonlinear interactions beyond the scope of our linear approximation. In particular, it is not possible to explain why 'Mode $\mathrm{B}$ ' is observed experimentally below $R e_{2}^{\prime}$. We stress, however, that the transition from 'Mode A' to 'Mode B' is tied to the linear 
stability of 'Mode $A$ ' and not directly to the linear stability of the two-dimensional wake. It is not inconsistent that 'Mode A' undergoes transition to 'Mode B' prior to $R e_{2}^{\prime}$. Furthermore, the existence of a three-dimensional flow with non-zero $\lambda$ implies the existence of smaller scales generated by nonlinearity: once 'Mode $A$ ' is established, the three-dimensional flow necessarily includes wavelengths in the 'Mode B' range.

The differences between 'Mode A' and 'Mode B' considered at the end of $\$ 3$ are consistent with the deductions of Williamson $(1996 c)$ in which he finds that the two modes observed experimentally have different symmetries and speculates that they arise from different physical mechanisms. In particular he suggests that 'Mode $A$ ' is an instability of the vortex cores, while the observed wavelength of 'Mode B' indicates an instability of the 'braid' regions. This is clearly consistent with the structure of the two Floquet modes shown in figure 11 . Vorticity modes with these same symmetries were also found in earlier studies of three-dimensionality in plane wakes by Meiburg \& Lasheras (1988) and Lasheras \& Meiberg (1990).

Direct numerical simulations of transition performed by Thompson, Hourigan \& Sheridan $(1994,1996)$ support the possibility of co-existing modes with different length scales and provide additional qualitative agreement with the linear growth rates predicted by our calculations. At $R e=250$, they report that the first organized three-dimensional structure to appear from a low-amplitude, broad-band disturbance has a wavelength of $\lambda=\pi$ diameters (the longest wavelength included in their calculation); only after this state has developed does a new structure emerge with a shorter wavelength of $\lambda \approx 1$ diameter. Even in this final state, visualization of the three-dimensional flow shows that the streamwise vortex spacing is $\lambda / d \approx 1$ in the near wake while $\lambda / d \approx \pi$ appears further downstream. DNS calculations by Zhang $e t$ al. (1995) show similar length scales at this Reynolds number. However, neither of these studies reports growth rates during the transition between two-dimensional and three-dimensional states that we might compare to directly.

Limited experimental data is available for comparison over a wider range of Reynolds number. Mansy, Yang \& Williams (1994) have made accurate measurements of spanwise wavelengths using a scanning laser anemometer to extract velocity maps of the near wake. They find that $\lambda \approx 3$ to 4 diameters for $180<R e<240$, with a shorter wavelength, $\lambda \approx 1$ diameter, appearing above Reynolds number 240 . Most of their long-wavelength data above $R e=188$ fall within the upper unstable region in our stability diagram (labelled ' $A$ ' in figure 12), and all of their short-wavelength data between $R e=260$ and $R e=300$ fall within the lower unstable region (labelled ' $B$ ' in figure 12). Measurements by $\mathrm{Wu}$ et al. (1994) using hydrogen bubble flow visualization all fall in the lower unstable region for $R e>260$.

While the issue of wavenumber selection after transition is beyond the scope of a linear analysis, we note the following. The general trend reported by Mansy et al. (1994) is that wavelength decreases with Reynolds number as $\lambda / d \sim R e^{-i / 2}$. In the range of Reynolds number reported here there is a more subtle variation. For 'Mode A', experimental values of $\lambda$ are very near the short-wavelength side of the 'A' band. In contrast, measurements for 'Mode B' tend to lie near the long-wavelength side of the 'B' band. The point of maximum growth rate of linear modes shifts to shorter wavelengths (larger wavenumbers) with increasing Reynolds number for the unstable modes corresponding to 'Mode $\mathrm{A}$ ' in figure 5, whereas the point of maximum growth rate shifts to longer wavelengths (smaller wavenumbers) with increasing Reynolds number for the 'Mode B' unstable modes in figure 7. In both cases, the point of maximum growth rate is asymmetrically located within the unstable bands in a way 
consistent with the experimental trends. It is not clear at what precise value of $R e$ the trend reported by Mansy et al. begins.

The development of streamwise vorticity in the wake is a striking feature of the three-dimensional flow discussed at some length by a number of researchers (e.g. Williamson 1988, 1996a,b; Wu et al. 1994, 1996; Mittal \& Balachandar 1995). During transition, the presence of streamwise vorticity is simply a consequence of the three-dimensional structure of the Floquet mode driving the instability; it may be more appropriate to discuss simply the three-dimensional vorticity field of the perturbation. Experimental flow visualization clearly shows the presence of a strong three-dimensional vorticity field downstream of the cylinder. This is linked to the growth in amplitude of the global mode (Floquet mode) controlling the threedimensional structure of the flow. Our computations show that all three vorticity components are generated very close to the cylinder, and in fact originate inside the recirculation zone. This underscores the fact that the development of threedimensionality in the wake is driven by a near-wake absolute instability.

The numerical studies discussed above (Thompson et al. 1994, 1996; Zhang et al. 1995) have managed to reproduce the long- and short-wavelength instabilities predicted by our calculations and observed in experiments. Direct numerical simulations will no doubt serve to improve our understanding of the physical mechanisms behind these instabilities. However, simulations are problematic in that they restrict the flow dynamics by imposing spanwise periodicity. Periodic boundary conditions reduce the flow to a discrete set of spanwise wavenumbers, in a sense mimicking a closed system with a new fundamental length scale: the periodic length. Computational results depend strongly on the imposed periodic length. In particular, the original study by Karniadakis \& Triantafyllou (1992) was for a spanwise length of $L=\pi / 2$ diameters. The effect such restrictions has on the wake dynamics is not clear, and it is difficult to draw quantitative conclusions from the three-dimensional simulations.

\subsection{Beyond the secondary instability}

We conclude with some remarks on subsequent bifurcations and in particular on possible scenarios leading to chaos in the cylinder wake. Because no new temporal frequency is introduced by the secondary instability, and because the only symmetry broken is translational symmetry along the cylinder axis, the bifurcation to a threedimensional flow would appear to be simple from a dynamical systems viewpoint. Thus a natural question is: "What is the scenario leading to complex dynamics in the cylinder wake?"

Numerical studies by Karniadakis \& Triantafyllou (1992) and Tomboulides et al. (1992) suggest that further increases in $R e$ result in a gradual sequence of period-doubling bifurcations leading to chaos. These bifurcations are marked by the appearance of subharmonic fluctuations in the wake. In the context of perioddoubling, the term subharmonic refers to fluctuations in time over the set of frequencies $f / 2^{n}$ for integers $n>0$, where $f$ is the dominant vortex shedding frequency. If this is the case, it should be possible to measure experimentally a clean progression of subharmonics as each period-doubling occurs. Recent measurements by Williams, Mansy \& Abouel-Fotouh (1996) have identified a subharmonic fluctuation at $R e=$ 300 , but have not established that this forms the beginning of a period-doubling cascade. Other experimentally measured frequency spectra (e.g. Williamson 1988, 1989; Leweke \& Provansal 1994, 1995) are, in fact, broad-band at Reynolds numbers just above $\operatorname{Re}_{2}$. This would suggest to the contrary that the flow becomes complex at the onset of three-dimensionality. 
Leweke \& Provansal $(1994,1995)$ have proposed a transition scenario, based on the complex Ginzburg-Landau equation, that captures the experimental spectra quite well and supports intrinsically complex dynamics just beyond the secondary instability. However, in this scenario the secondary instability is of the Benjamin-Feir type (a zerowavenumber instability) while both our computations and experimental observations show that the instability occurs at a finite spanwise wavenumber. While it is intriguing that a simple nonlinear model equation may capture the essential dynamical features of this complex flow, the scenario proposed by Leweke \& Provansal lacks a finitewavenumber instability and some modification of their model is necessary.

The question remains open as to whether the appearance of complex dynamics in the cylinder wake is gradual or abrupt, i.e. are the broad-band spectra seen in experiments the result of intrinsically complex (chaotic) dynamics in the wake at Reynolds numbers just beyond the secondary instability, or are they due to small, random fluctuations? Accurate knowledge of the linear stability properties of the wake should allow future studies, either computational or experimental, to address this question with greater precision than has been possible in the past. This and other interesting questions about the wake dynamics beyond the secondary instability remain to be answered.

We wish to thank H. M. Blackburn, M. Gharib, A. Leonard, T. Leweke, D. Meiron, B. R. Noack, A. Roshko, L. S. Tuckerman, D. R. Williams, and C. H. K Williamson for their questions, comments, and suggestions which have added significantly to this paper. D. B. acknowledges the hospitality of the Center for Nonlinear Dynamics at the University of Texas at Austin where part of this work was conducted. He also thanks the Nuffield Foundation for their support and the NSF for their support though Grant No. DMS 92-06224. R. D. H. acknowledges financial support from the NSF through Grant No. CDA-9318145 and the ONR through Grant No. N000-94-1-0793. Computational resources for this work were provided by the University of Texas System Center for High Performance Computing and the JPL High Performance Computing and Communications program at the California Institute of Technology.

\section{REFERENCES}

Amon, C. H. \& Patera, A. T. 1989 Numerical calculation of stable three-dimensional tertiary states in grooved-channel flow. Phys. Fluids A 1, 2005-2009.

BARKLEY, D. 1990 Theory and predictions for finite-amplitude waves in 2-dimensional plane Poiseuille flow. Phys. Fluids A 2, 955-970.

Goldhirsch, I., OrszaG, S. A. \& Maulik, B. K. 1987 An efficient method for computing leading eigenvalues and eigenvectors of large asymmetric matrices. J. Sci. Comput. 2, 33-58.

Guckenheimer, J. \& Holmes, P. 1983 Nonlinear Oscillations, Dynamical Systems, and Bifurcations of Vector Fields. Springer.

Hammache, M. \& Gharib, M. 1991 An experimental study of the parallel and oblique vortex shedding from circular cylinders. J. Fluid Mech. 232, 567-590.

Henderson, R. D. 1994 Unstructured Spectral Element Methods: Parallel Algorithms and Simulations. $\mathrm{PhD}$ thesis Princeton University.

Henderson, R. D. \& BarkLey, D. 1996 Secondary instability in the wake of a circular cylinder. Phys. Fluids 8, 1683-1685.

Henderson, R. D. \& Karniadakis, G. E. 1995 Unstructured spectral element methods for simulation of turbulent flows. J. Comput. Phys. 122, 191-217.

Huerre, P. \& Monkewitz, P. A. 1990 Local and global instabilities in spatially developing flows. Ann. Rev. Fluid Mech. 22, 473-537. 
JACKSON, C. P. 1987 A finite-element study of the onset of vortex shedding in flow past variously shaped bodies. J. Fluid Mech. 182, 23-45.

Karniadakis, G. E., Israeli, M. \& OrszaG, S. A. 1991 High-order splitting methods for the incompressible Navier-Stokes equations. J. Comput. Phys. 97, 414413.

Karniadakis, G. E. \& Triantafyllou, G. S. 1989 Frequency selection and asymptotic states in laminar wakes. J. Fluid Mech. 199, 441-469.

Karniadakis, G. E. \& Triantafyllou, G. S. 1992 Three-dimensional dynamics and transition to turbulence in the wake of bluff objects. J. Fluid Mech. 238, 1-30.

LASHERAS, J. C. \& MEIBURG, E. 1990 Three-dimensional vorticity modes in the wake of a flat plate. Phys. Fluids A 2, 371-380.

Leweke, T. \& Provansal, M. 1994 Model for the transition in bluff-body wakes. Phys. Rev. Lett. 72, 3174-3177.

LeWeke, T. \& Provansal, M. 1995 The flow behind rings - bluff-body wakes without end effects. J. Fluid Mech. 288, 265-310.

Mamun, C. K. \& Tuckerman, L. S. 1995 Asymmetry and Hopf bifurcation in spherical Couette flow. Phys. Fluids 7, 80 91.

MaNSY, H., YANG, P.-M. \& Williams, D. R. 1994 Quantitative measurements of three-dimensional structures in the wake of a circular cylinder. J. Fluid Mech. 270, 277-296.

Mathis, C., Provansal, M. \& Boyer, L. 1987 Bénard-von Kármán instability: transient and forced regimes. J. Fluid Mech. 182, 1-22.

Meiburg, E. \& Lasheras, J. C. 1988 Experimental and numerical investigation of the threedimensional transition in plane wakes. J. Fluid Mech. 190, 1-37.

Miller, G. D. \& Williamson, C. H. K. 1994 Control of three-dimensional phase dynamics in a cylinder wake. Exps. Fluids 18, 26-35.

Mittal, R. \& Balachandar, S. 1995 Generation of streamwise vortical structures in bluff body wakes. Phys. Rev. Lett. 75, 1300-1303.

Noack, B. R. \& Eckelmann, H. 1994a A global stability analysis of the steady and periodic cylinder wake. J. Fluid Mech. 270, 297-330.

Noack, B. R. \& Eckelmann, H. $1994 b$ A low-dimensional Galerkin method for the threedimensional flow around a circular cylinder. Phys. Fluids A 6, 124-143.

NoACK, B. R., König, M. \& ECKelmanN, H. 1993 Three-dimensional stability analysis of the periodic flow around a circular cylinder. Phys. Fluids A 5, 1279-1281.

NORBERG, C. 1994 An experimental investigation of the flow around a circular cylinder: influence of aspect ratio. J. Fluid Mech. 258, 287-316.

Roshko, A. 1993 Perspectives on bluff body aerodynamics. J. Wind Engng 49, 79-100.

SCHATZ, M. F., BarkLEY, D. \& SwinNey, H. L. 1995 Instability in spatially periodic open flow. Phys. Fluids 7, 344-358.

Thompson, M., Hourigan, K. \& Sheridan, J. 1994 Three-dimensional instabilities in the cylinder wake. In Intl Colloq. Jets, Wakes, and Shear Layers. Melbourne, Australia. CSIRO.

Thompson, M., Hourigan, K. \& Sheridan, J. 1996 Three-dimensional instabilities in the wake of a circular cylinder. Exp. Therm. Fluid Sci. 12, 190-196.

Tomboulides, A. G., Triantafyllou, G. S. \& Karniadakis, G. E. 1992 A new mechanism of period doubling in free shear flows. Phys. Fluids A 4, 1329-1332.

Watkins, D. S. 1993 Some perspectives on the eigenvalue problem. SIAM Rev. 35, 430-471.

Williams, D. R., Mansy, H. \& Abolel-Fotouh, A. 1996 Three-dimensional subharmonic waves during transition in the near-wake region of a cylinder. Phys. Fluids 8, 1476-1485.

Williamson, C. H. K. 1988 The existence of two stages in the transition to three dimensionality of a cylinder wake. Phys. Fluids 31, 3165-3168.

Williamson, C. H. K. 1989 Oblique and parallel modes of vortex shedding in the wake of a circular cylinder at low Reynolds numbers. J. Fluid Mech. 206, 579-627.

Williamson, C. H. K. 1992 The natural and forced formation of spot-like 'vortex dislocations' in the transition of a wake. J. Fluid Mech. 243, 393-441.

Williamson, C. H. K. 1996a "Mode A" secondary instability in wake transition. Phys. Fluids 8 , $1680-1682$.

Williamson, C. H. K. $1996 b$ Vortex dynamics in the cylinder wake. Ann. Rev. Fluid Mech. 28. 477-539. 
Williamson, C. H. K. 1996c Three-dimensional transition in the cylinder wake. J. Fluid Mech. (to appear).

Williamson, C. H. K. \& Roshko, A. 1990 Measurements of base pressure in the wake of a cylinder at low Reynolds numbers. Z. Flugwiss. Weltraumforsch. 14, 38-46.

Wu, J., Sheridan, J., Soria, J. \& Welsh, M. C. 1994 An experimental investigation of streamwise vortices in the wake of a bluff body. J. Fluid Struct. 8, 621-625.

Wu, J., Sheridan, J., Welsh, M. C. \& Hourigan, K. 1996 Three-dimensional vortex structures in a cylinder wake. J. Fluid Mech. 312, 201-222

Zhang, H.-Q., Fey, U., Noack, B. R., König, M. \& Eckelmann, H. 1995 On the transition of the cylinder wake. Phys. Fluids 7, 779-794. 